1

2

3

\title{
Minimum Augmentation of Edge-Connectivity between Vertices and Sets of Vertices in Undirected Graphs*
}

\author{
Toshimasa Ishii ${ }^{\dagger}$, Yoko Akiyama ${ }^{\dagger \dagger}$, and Hiroshi Nagamochi ${ }^{\dagger \dagger}$ \\ ${ }^{\dagger}$ Department of Information and Management Science \\ Otaru University of Commerce, \\ Hokkaido 047-8501, Japan \\ E-mail: ishii@res.otaru-uc.ac.jp \\ ${ }^{\dagger \dagger}$ Allied Telesis K.K., Tokyo 141-0031, Japan \\ ${ }^{\dagger \dagger}$ Department of Applied Mathematics and Physics, \\ Graduate School of Informatics, Kyoto University, \\ Kyoto 606-8501, Japan \\ E-mail: nag@amp.i.kyoto-u.ac.jp
}

\author{
Corresponding Author: Toshimasa Ishii \\ Department of Information and Management Science \\ Otaru University of Commerce, Hokkaido 047-8501, Japan \\ Phone\&Fax: +81-134-27-5389 \\ E-mail: ishii@res.otaru-uc.ac.jp
}

Keywords: undirected graph, connectivity augmentation problem, edge-connectivity, node-toarea connectivity, polynomial time deterministic algorithm, edge-splitting.

\begin{abstract}
Given an undirected multigraph $G=(V, E)$, a family $\mathcal{W}$ of areas $W \subseteq V$, and a target connectivity $k \geq 1$, we consider the problem of augmenting $G$ by the smallest number of new edges so that the resulting graph has at least $k$ edge-disjoint paths between $v$ and $W$ for every pair of a vertex $v \in V$ and an area $W \in \mathcal{W}$. So far this problem was shown to be NP-complete in the case of $k=1$ and polynomially solvable in the case of $k=2$. In this paper, we show that the problem for $k \geq 3$ can be solved in $O\left(m+n\left(k^{3}+n^{2}\right)(p+k n+n \log n) \log k+p k n^{3} \log (n / k)\right)$ time, where $n=|V|, m=|\{\{u, v\} \mid(u, v) \in E\}|$, and $p=|\mathcal{W}|$.
\end{abstract}

\footnotetext{
*An extended abstract of this paper was presented at 9th Computing: The Australasian Theory Symposium (CATS 2003), Australia, February 2003.
} 


\section{Introduction}

In a communication network, graph connectivity is a fundamental measure of its robustness. An undirected graph $G=(V, E)$ is $k$-edge-connected if the deletion of any $k-1$ or fewer edges leaves a connected graph; equivalently, there exist at least $k$ pairwise edge-disjoint paths between every two vertices. The connectivity augmentation problem asks to add to a given graph the smallest number of new edges such that the connectivity of the graph increases up to a specified value $k$. The problem has important applications such as the network design problem [8], the rigidity problem in grid frameworks [3], the data security problem [14], the rectangular dual graph problem in floor-planning [21], and the graph drawing problem [13], and many efficient algorithms have been developed so far.

Most of all those researches have dealt with connectivity between two vertices in a graph. However, in many real-wold networks, the connectivity between every two vertices is not necessarily required. For example, in a multimedia network, some vertices of the network may have functions of offering several types of services for users. For a set $W$ of vertices offering certain service $i$, a user at a vertex $v$ can use service $i$ by communicating with one vertex $w \in W$ through a path between $w$ and $v$. In such networks, it is desirable that the network has some pairwise disjoint paths from the vertex $v$ to at least one of vertices in $W$. This means that the measure of reliability is the connectivity between a vertex and a set of vertices rather than that between two vertices. From this point of view, H. Ito et al. considered the node to area connectivity (NA-connectivity, for short) as a concept that represents the connectivity between vertices and sets of vertices (areas) in a graph $[9,10,12]$. As related problems, the problem of locating a set $W$ of vertices offering service with requirements measured by connectivity has been also studied $[1,11,12,19]$.

In this paper, given a graph $G=(V, E)$ with a family $\mathcal{W}$ of areas $W \subseteq V$, and a positive integer $k$, we consider the problem of asking to augment $G$ by adding the smallest number of new edges so that the resulting graph has at least $k$ pairwise edge-disjoint paths between $v$ and $W$ for every pair of a vertex $v \in V$ and an area $W \in \mathcal{W}$. We call this problem $k$-NA-edge-connectivity augmentation problem (for short, $k-N A-E C A P$ ). Figure 1 gives an instance of 3-NA-ECAP. In the graph $G$ in (i), some pair of a vertex $v \in V$ and an area $W \in \mathcal{W}$ (say, $v_{8}$ and $W_{3}$ ) cannot have three edge-disjoint paths between them, and 3-NA-ECAP asks to add the minimum number of new edges to $G$ to construct a graph like (ii) in which there are at least three edge-disjoint paths between every pair of $v \in V$ and $W \in \mathcal{W}$. H. Miwa et al. [15] showed that 1-NA-ECAP is NP-hard by a reduction from SET SPLITTING.

Lemma 1.1 [15] 1-NA-ECAP is NP-hard. 
Figure 1: Illustration of an instance of 3-NA-ECAP. (i) An initial graph $G=(V, E)$ with a family $\mathcal{W}=\left\{W_{1}, W_{2}, W_{3}\right\}$ of areas. (ii) A 3-NA-edge-connected graph obtained from $G$ by adding a set of edges drawn as broken lines; there are at least three edge-disjoint paths between every pair of a vertex $v \in V$ and an area $W \in \mathcal{W}$.

Proof SKETCH: SET SPLITTING is reduced to 1-NA-ECAP:

\section{SET SPLITTING [4]}

Instance: A family $\mathcal{C}$ of subsets of a finite ground set $S$.

Objective: Find a bipartition $\left\{S_{1}, S_{2}\right\}$ of $S$ such that no subset in $C$ is entirely included in either $S_{1}$ or $S_{2}$.

Given an instance of SET SPLITTING, we construct an instance of 1-NA-ECAP as follows: Let $G=(S, \emptyset)$ and $\mathcal{W}=\mathcal{C}$. Then we can prove that $G$ can be augmented by adding at most $|S|-2$ edges if and only if $S$ has a feasible bipartition. Indeed, if there is a feasible bipartition $\left\{S_{1}, S_{2}\right\}$ of $S$ to SET SPLITTING, then the union of an edge set spanning $S_{1}$ and that spanning $S_{2}$ is a solution to 1-NA-ECAP in $G$. If there is a solution $E^{\prime}$ with cardinality at most $|S|-2$ to 1-NA-ECAP, then the augmented graph $\left(S, E^{\prime}\right)$ is disconnected and each component in $\left(S, E^{\prime}\right)$ does not include any subset in $\mathcal{C}$, which implies that a feasible bipartition of $S$ exists.

They also showed that 2-NA-ECAP can be solved in polynomial time. However, it was still open whether the problem in the case of $k \geq 3$ is polynomially solvable or not.

Notice that if some area $W \in \mathcal{W}$ satisfies $|W|=1$, then $k$-NA-ECAP is equivalent to the classical $k$-edge-connectivity augmentation problem (for short, $k$-ECAP) which augments the edge-connectivity of a given graph. Indeed, a graph has pairwise $k$ edge-disjoint paths between each pair of a vertex and an area for any given family of areas if it is $k$-edge-connected, and a graph is $k$-edge-connected if it has $k$ pairwise edge-disjoint paths between one fixed vertex and any other vertex. It was shown that $k$-ECAP is polynomially solvable by $\mathrm{T}$. Watanabe et al. [22] and A. Frank[5]. Many algorithms for $k$-ECAP have been studied [5, 6, 7, 18, 22]. In [5], A. Frank also solved a more general problem such as local edge-connectivity augmentation 


\section{Problem Definition}

Let $G=(V, E)$ stand for an undirected graph with a set $V$ of vertices and a set $E$ of edges. An edge with end vertices $u$ and $v$ is denoted by $(u, v)$. We denote $|V|$ by $n$ and $|\{\{u, v\} \mid(u, v) \in E\}|$ by $m$. A singleton set $\{x\}$ may be simply written as $x$, and " $\subset$ " implies proper inclusion while " $\subseteq$ " means " $\subset$ " or " $=$ ". In $G=(V, E)$, its vertex set $V$ and edge set $E$ may be denoted by $V(G)$ and $E(G)$, respectively. For a subset $V^{\prime} \subseteq V$ in $G, G\left[V^{\prime}\right]$ denotes the subgraph induced by $V^{\prime}$. For an edge set $E^{\prime}$ with $E^{\prime} \cap E=\emptyset$, we denote the augmented graph $\left(V, E \cup E^{\prime}\right)$ by $G+E^{\prime}$. For an edge set $E^{\prime}$, we denote by $V\left[E^{\prime}\right]$ the set of all end vertices of edges in $E^{\prime}$.

An area graph is defined as a graph $G=(V, E)$ with a family $\mathcal{W}$ of vertex subsets $W \subseteq V$ which are called areas (see Figure 1$)$. We denote an area graph $G$ with $\mathcal{W}$ by $(G, \mathcal{W})$. In the sequel, we may denote $(G, \mathcal{W})$ simply by $G$ if no confusion arises. For two disjoint subsets $X, Y \subset V$ of vertices, we denote by $E_{G}(X, Y)$ the set of edges $e=(x, y)$ such that $x \in X$ and $y \in Y$, and also denote $\left|E_{G}(X, Y)\right|$ by $d_{G}(X, Y)$. In particular, $E_{G}(u, v)$ is the set of edges with end vertices $u$ and $v$. A cut is defined as a subset $X$ of $V$ with $\emptyset \neq X \neq V$, and the size of a cut $X$ is defined by $d_{G}(X, V-X)$, which may also be written as $d_{G}(X)$. Moreover, we define $d(\emptyset)=0$. For two cuts $X, Y \subseteq V$ in a graph $G=(V, E)$, we say that $X$ and $Y$ cross each other in $G$ or $X$ crosses with $Y$ if none of $X \cap Y, X-Y, Y-X$, and $V-(X \cup Y)$ is empty. For a 
For two cuts $X, Y \subset V$ with $X \cap Y=\emptyset$ in $G$, we denote by $\lambda_{G}(X, Y)$ the minimum size of cuts which separate $X$ and $Y$, i.e., $\lambda_{G}(X, Y)=\min \left\{d_{G}(S) \mid S \supseteq X, S \subseteq V-Y\right\}$. For two cuts $X, Y \subset V$ with $X \cap Y \neq \emptyset$ in $G$, we define $\lambda_{G}(X, Y)=\infty$. The edge-connectivity of $G$, denoted by $\lambda(G)$, is defined as $\min _{X \subset V, Y \subset V} \lambda_{G}(X, Y)$. For a vertex $x \in V$ and a set $W \subseteq V$ of vertices, the node-to-area edge-connectivity (NA-edge-connectivity, for short) between $x$ and $W$ is defined as $\lambda_{G}(x, W)$. Note that $\lambda_{G}(x, W)=\infty$ holds for $x \in W$. For an area graph $(G, \mathcal{W})$, the $N A$-edge-connectivity of $G$, denoted by $\lambda(G, \mathcal{W})$, is defined as $\min _{x \in V, W \in \mathcal{W}} \lambda_{G}(x, W)$. Note that the area graph $(G, \mathcal{W})$ in Figure $1(\mathrm{i})$ satisfies $\lambda(G, \mathcal{W})=1$. If $\lambda(G, \mathcal{W}) \geq k$ holds, then we say that $(G, \mathcal{W})$ is $k$-NA-edge-connected.

In this paper, we consider the following problem, called $k$-NA-ECAP.

Problem 2.1 ( $k$-NA-edge-connectivity augmentation problem, $k$-NA-ECAP) Input: An area graph $(G=(V, E), \mathcal{W})$ and a positive integer $k$. Output: A set $E^{*}$ of new edges with $\lambda\left(G+E^{*}, \mathcal{W}\right) \geq k$ such that $\left|E^{*}\right|$ is the minimum.

\section{Lower Bounds on the Optimal Value}

For an area graph $(G, \mathcal{W})$ and a fixed integer $k$, let $\operatorname{opt}_{k}(G, \mathcal{W})$ denote the optimal value to $k$-NA-ECAP in $G$, i.e., the minimum size $\left|E^{*}\right|$ of a set $E^{*}$ of new edges such that $G+E^{*}$ is $k$-NA-edge-connected. In this section, we derive a lower bound on $\operatorname{opt}_{k}(G, \mathcal{W})$ to $k$-NA-ECAP with $(G, \mathcal{W})$.

A family $\mathcal{X}=\left\{X_{1}, \ldots, X_{t}\right\}$ of cuts in $G$ is called a partition of $V$, if every two cuts $X_{i}, X_{j} \in \mathcal{X}$ satisfy $X_{i} \cap X_{j}=\emptyset$ and $\cup_{X \in \mathcal{X}} X_{i}=V$ holds. For a subset $X \subseteq V$ of vertices, a partition of $X$ is called a subpartition of $V$. In an area graph $(G, \mathcal{W})$, a cut $X$ is called type $(A)$ if $X \cap W=\emptyset$ holds for some area $W \in \mathcal{W}$, and a cut $X$ is called type $(B)$ if $X \supseteq W$ holds for some area $W \in \mathcal{W}$ (note that a cut $X$ of type (B) satisfies $X \neq V$ by the definition of a cut). Note that $X$ is of type (A) if and only if $V-X$ is of type (B), and that each cut can be of both types.

Let $p(X)=\max \left\{0, k-d_{G}(X)\right\}$ for each cut $X$ of type (A) or (B) and $p(X)=0$ for all other subsets $X \subseteq V$. We easily see the following property.

Lemma 3.1 An area graph $(G, \mathcal{W})$ satisfies $\lambda(G, \mathcal{W}) \geq k$ if and only if $d_{G}(X) \geq k$ holds for every cut $X \subset V$ of type $(A)$ or $(B) ; p(X)=0$ for all cuts $X$.

For each cut $X$ of type (A) or (B) with $d_{G}(X)<k$, it is necessary to add at least $k-d_{G}(X)$ edges between $X$ and $V-X$. Thus if $X$ is of type (A) (resp., type (B)), then the NA-edge- 
connectivity between a vertex in $X$ (resp., $V-X$ ) and an area $W \in \mathcal{W}$ with $W \cap X=\emptyset$ (resp., $W \subseteq X)$ need be augmented to at least $k$.

Let

$$
\alpha_{k}(G, \mathcal{W})=\max _{\mathcal{X}}\left\{\sum_{X \in \mathcal{X}} p(X)\right\}
$$

where the maximization is taken over all subpartitions of $V$. Then any feasible solution to $k$-NA-ECAP with $(G, \mathcal{W})$ must contain an edge which joins two vertices from a cut $X$ of type (A) or (B) and the cut $V-X$. Thus we have the following lemma.

Lemma $3.2 \operatorname{opt}_{k}(G, \mathcal{W}) \geq\left\lceil\alpha_{k}(G, \mathcal{W}) / 2\right\rceil$ holds.

The area graph $(G, \mathcal{W})$ in Figure $1(\mathrm{i})$ satisfies $\alpha_{3}(G, \mathcal{W})=10$. We have $\sum_{X \in \mathcal{X}} p(X)=\sum_{X \in \mathcal{X}}(3-$ $\left.d_{G}(X)\right)=10$ for the subpartition $\mathcal{X}=\left\{\left\{v_{1}\right\},\left\{v_{2}\right\},\left\{v_{3}\right\},\left\{v_{4}\right\},\left\{v_{5}\right\},\left\{v_{6}\right\},\left\{v_{7}\right\},\left\{v_{8}, v_{9}\right\}\right\}$ of $V$.

In the classical $k$-ECAP, which is a special case of $k$-NA-ECAP, it is known that this type of lower bound based on subpartitions of $V$ is equal to the optimal value [5, 22]. However, in $k$-NA-ECAP, there are area graphs $(G, \mathcal{W})$ with $\operatorname{opt}_{k}(G, \mathcal{W})>\left\lceil\alpha_{k}(G, \mathcal{W}) / 2\right\rceil$. Figure 2 gives an instance for $k=2$. Each cut $\left\{v_{i}\right\}$ is of type (A) satisfies $p\left(v_{i}\right)=k-d_{G}\left(v_{i}\right)=1$ for $i=1,2,3,4$. Thus $\left\lceil\alpha_{2}(G, \mathcal{W}) / 2\right\rceil=2$. In order to make $(G, \mathcal{W})$ 2-NA-edge-connected by adding two new edges, we must add $e=\left(v_{1}, v_{2}\right)$ and $e^{\prime}=\left(v_{3}, v_{4}\right)$ without loss of generality. $G+\left\{e, e^{\prime}\right\}$ is not 2-NA-edge-connected by $\lambda_{G+\left\{e, e^{\prime}\right\}}\left(v_{3}, W_{1}\right)=1$. We will show that all such instances can be

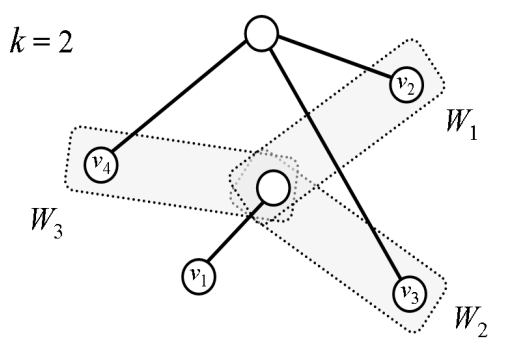

Figure 2: Illustration of an area graph $(G, \mathcal{W})$ with $\operatorname{opt}_{2}(G, \mathcal{W})=\left\lceil\frac{\alpha_{2}(G, \mathcal{W})}{2}\right\rceil+1$.

completely characterized as follows.

Definition 3.3 We say that an area graph $(G, \mathcal{W})$ has property $(\mathrm{P})$ if $\alpha_{k}(G, \mathcal{W})$ is even and there is a subpartition $\mathcal{X}=\left\{X_{1}, \ldots, X_{q}\right\}$ of $V$ with $\sum_{X \in \mathcal{X}} p(X)=\alpha_{k}(G, \mathcal{W})$ satisfying the following conditions (P1)-(P3):

(P1) Each cut $X_{i} \in \mathcal{X}$ is of type (A).

(P2) The cut $X_{1}$ satisfies $d_{G}\left(X_{1}\right)=k-1$ and $X_{1} \subset C_{1}$ for some component $C_{1}$ of $G$ with $X_{i} \cap C_{1}=\emptyset$ for each $i=2, \ldots, q$. 
(P3) For each $i=2, \ldots, q$, there is a cut $Y_{i}$ of type (B) with $X_{i} \cup X_{1} \subseteq Y_{i}$ and $\sum_{X \in \mathcal{X}, X \subset Y_{i}}(k-$ $\left.d_{G}(X)\right) \leq(k+1)-d_{G}\left(Y_{i}\right)$ such that every cut $X \in \mathcal{X}$ satisfies $X \subset Y_{i}$ or $X \cap Y_{i}=\emptyset$.

Note that $(G, \mathcal{W})$ in Figure 2 has property $(\mathrm{P})$ because $\alpha_{2}(G, \mathcal{W})=4$ holds and the subpartition $\mathcal{X}$ of $V$ consisting of $X_{i}=\left\{v_{i}\right\}, i=1,2,3,4$ satisfies $Y_{2}=C_{1} \cup\left\{v_{2}\right\}, Y_{3}=C_{1} \cup\left\{v_{3}\right\}$, and $Y_{4}=C_{1} \cup\left\{v_{4}\right\}$ for the component $C_{1}$ of $G$ containing $v_{4}$.

Lemma 3.4 If $(G, \mathcal{W})$ has property $(\mathrm{P})$, then opt $(G, \mathcal{W}) \geq\left\lceil\alpha_{k}(G, \mathcal{W}) / 2\right\rceil+1$ holds.

Proof: Assume by contradiction that there is an edge set $E^{*}$ with $\lambda\left(G+E^{*}, \mathcal{W}\right) \geq k$ and $\left|E^{*}\right|=\alpha_{k}(G, \mathcal{W}) / 2$ (note that $\alpha_{k}(G, \mathcal{W})$ is even). Let $\mathcal{X}=\left\{X_{1}, \ldots, X_{q}\right\}$ denote a subpartition of $V$ satisfying $\sum_{X \in \mathcal{X}} p(X)=\alpha_{k}(G, \mathcal{W})$ and the above $(\mathrm{P} 1)-(\mathrm{P} 3)$. Since $\left|E^{*}\right|=\alpha_{k}(G, \mathcal{W}) / 2$ holds, each cut $X \in \mathcal{X}$ satisfies $d_{G+E^{*}}(X)=k$, and hence $d_{G^{\prime}}(X)=k-d_{G}(X)$, where $G^{\prime}=\left(V, E^{*}\right)$. Therefore, any edge $\left(x_{i}, x_{j}\right) \in E^{*}$ satisfies $x_{i} \in X_{i}$ and $x_{j} \in X_{j}$ for some two cuts $X_{i}, X_{j} \in \mathcal{X}$ with $X_{i} \neq X_{j}$. From this, there exists a cut $X_{\ell} \in \mathcal{X}$ with $\ell \neq 1$ and $E_{G^{\prime}}\left(X_{\ell}, X_{1}\right) \neq \emptyset$. Since $(G, \mathcal{W})$ satisfies property $(\mathrm{P})$, there is a cut $Y_{\ell}$ which satisfies $(\mathrm{P} 3)$, and hence $\sum_{v \in Y_{\ell}} d_{G^{\prime}}(v) \leq$ $(k+1)-d_{G}\left(Y_{\ell}\right)$. Since $G^{\prime}\left[Y_{\ell}\right]$ contains one edge in $E_{G^{\prime}}\left(X_{\ell}, X_{1}\right)$, we have $d_{G^{\prime}}\left(Y_{\ell}\right) \leq(k-1)-d_{G}\left(Y_{\ell}\right)$, which implies that $G+E^{*}$ is not $k$-NA-edge-connected, a contradiction.

In this paper, we prove that $k$-NA-ECAP enjoys the following min-max theorem and is polynomially solvable.

Theorem 3.5 For $k$-NA-ECAP with $k \geq 3$ opt $_{k}(G, \mathcal{W})=\left\lceil\alpha_{k}(G, \mathcal{W}) / 2\right\rceil$ holds if $(G, \mathcal{W})$ does not have property $(P)$, and $\operatorname{opt}_{k}(G, \mathcal{W})=\left\lceil\alpha_{k}(G, \mathcal{W}) / 2\right\rceil+1$ holds otherwise. Moreover, a solution $E^{*}$ with $\left|E^{*}\right|=\operatorname{opt}_{k}(G, \mathcal{W})$ can be obtained in $O\left(m+n\left(n^{2}+k^{3}\right)(p+k n+n \log n) \log k\right)$ time.

\section{Extensions and Edge-Splittings}

\subsection{Extensions}

As mentioned in Section 1, we adapt the so-called "edge-splitting" method for solving $k$-NAECAP. In the edge-splitting method, after creating a new vertex $s$ outside of $G$ and adding new edges between $s$ and $G$, we find an appropriate edge set to be added to $G$ by splitting off a pair of edges incident to $s$ in the extended graph. Given an area graph $(G, \mathcal{W})$, a graph $H=(V \cup\{s\}, E \cup F)$ obtained from $(G, \mathcal{W})$ by adding a new vertex $s$ and a set $F$ of new edges connecting $s$ and $V$ is called a $k$-extension of $(G, \mathcal{W})$ if

$$
d_{H}(X) \geq k \text { holds for each cut } X \subset V \text { of type (A) or type (B). }
$$



violates (4.1) for any $F^{\prime} \subset F$. We here show that a critical $k$-extension $H=(V \cup\{s\}, E \cup F)$ of $(G, \mathcal{W})$ satisfies $|F|=\alpha_{k}(G)$.

Theorem 4.1 Let $(G=(V, E), \mathcal{W})$ be an area graph, and $k$ be a nonnegative integer. A critical $k$-extension $H=(V \cup\{s\}, E \cup F)$ of $(G, \mathcal{W})$ satisfies $|F|=\alpha_{k}(G, \mathcal{W})$.

In $(G, \mathcal{W})$, the following properties hold:

If a cut $X \subset V$ is of type $(A)$, then some area $W$ satisfies $W \cap X=\emptyset$ and hence every cut $X^{\prime} \subseteq X$ is also of type $(A)$.

If a cut $X \subset V$ is of type $(B)$, then some area $W$ satisfies $W \subseteq X$ and hence every cut $X^{\prime} \supseteq X$ with $X^{\prime} \neq V$ is also of type $(B)$.

Let $V$ be a finite ground set and let $q: 2^{V} \rightarrow Z^{+}$be an integer-valued function with $q(\emptyset)=0$, where $Z^{+}$denotes the set of nonnegative integers. A set function $q$ is called skew-supermodular if $q(X)+q(Y) \leq q(X \cap Y)+q(X \cup Y)$ or $q(X)+q(Y) \leq q(X-Y)+q(Y-X)$ hold for every two subsets $X$ and $Y$ of $V$ with $q(X)>0, q(Y)>0$. A set function $q$ is called symmetric if $q(X)=q(V-X)$ holds for all $X \subseteq V$. In [5, Section 7], [20, Lemma 5.1], it was shown that given a symmetric skew-supermodular integer-valued function $q: 2^{V} \rightarrow Z^{+}$, a function $z: V \rightarrow Z^{+}$ such that $\sum_{v \in V} z(v)$ is the minimum and $\sum_{v \in X} z(v) \geq q(X)$ holds for every $X \subseteq V$ can be found by a greedy algorithm. Now it is not difficult to see from (2.1), (2.2), (4.2), and (4.3) that $p$ is a symmetric skew-supermodular integer-valued function. Note that $H=(V \cup\{s\}, E \cup F)$ is a $k$-extension of $(G=(V, E), \mathcal{W})$ if and only if the function $z: V \rightarrow Z^{+}$with $z(v)=d_{H}(s, v)$ satisfies $z(X) \geq p(X)$ for every $X \subseteq V$. This observation proves Theorem 4.1.

Remarks: In [2], A. Benczúr and A. Frank considered a problem of covering a symmetric crossing supermodular function $q$ by the minimum number of edges, which is a generalization of $k$-ECAP, where a set function $q: 2^{V} \rightarrow Z^{+}$is called crossing supermodular if $q(X)+q(Y) \leq$ $q(X \cap Y)+q(X \cup Y)$ holds for every two crossing subsets $X$ and $Y$ of $V$ with $q(X)>0, q(Y)>0$. They gave a polynomial time algorithm for solving the problem, under the assumption that a polynomial oracle for $\min \left\{\sum_{v \in X} z(v)+d_{\left(V, E^{\prime}\right)}(X)-q(X) \mid X \subset V\right\}$ is given, where $z: V \rightarrow Z^{+}$ denotes a function on $V$ and $E^{\prime}$ denotes a set of edges. On the other hand, the function $p$ in $k$-NA-ECAP is not crossing supermodular. For example, in the graph in Figure 2, two cuts $X=C_{1} \cup\left\{v_{3}\right\}$ and $X^{\prime}=C_{1} \cup\left\{v_{4}\right\}$ satisfy $p(X)=p\left(X^{\prime}\right)=1$ and $p\left(X \cap X^{\prime}\right)=p\left(X \cup X^{\prime}\right)=0$, where $C_{1}$ denotes the component containing $v_{1}$ (note that $X \cap X^{\prime}=C_{1}$ is neither of type (A) nor of type (B)). 


\subsection{Edge-splitting theorems}

For a graph $H=(V \cup\{s\}, E)$ and a designated vertex $s \notin V$, an operation called edge-splitting (at $s$ ) is defined as deleting two edges $(s, u),(s, v) \in E$ and adding one new edge $(u, v)$. That is, the graph $H^{\prime}=(V \cup\{s\},(E-\{(s, u),(s, v)\}) \cup\{(u, v)\})$ is obtained from such edge-splitting operation. Then we say that $H^{\prime}$ is obtained from $H$ by splitting a pair of edges $(s, u)$ and $(s, v)$ (or by splitting $(s, u)$ and $(s, v)$ ). A sequence of splittings is complete if the resulting graph $H^{\prime}$ does not have any neighbor of $s$. The edge-splitting operation is known to be a useful tool for solving connectivity augmentation problems [5].

Given a $k$-extension $H=(V \cup\{s\}, E \cup F)$ of $(G, \mathcal{W})$, a pair $\{(s, u),(s, v)\}$ is called admissible if the graph $H^{\prime}$ obtained from $H$ by splitting $(s, u)$ and $(s, v)$ is also a $k$-extension of $H^{\prime}-s=$ $G+\{(u, v)\} ; H^{\prime}$ satisfies (4.1). Notice that given an area graph $(G, \mathcal{W})$, if there is a complete admissible splitting at $s$ in its critical $k$-extension $H=(V \cup\{s\}, E \cup F)$, then the set $E^{\prime}$ of split edges is an optimal solution of $k$-NA-ECAP to $(G, \mathcal{W})$. Indeed, every cut $X \subset V$ of type (A) or (B) satisfies $d_{G+E^{\prime}}(X) \geq k$, implying $\lambda\left(G+E^{\prime}, \mathcal{W}\right) \geq k$, since $H^{\prime}=\left(V \cup\{s\}, E \cup E^{\prime}\right)$ satisfies (4.1) and $d_{H^{\prime}}(s, V)=0$, and Theorem 4.1 implies that we have $\left|E^{\prime}\right|=|F| / 2=\left\lceil\alpha_{k}(G, \mathcal{W}) / 2\right\rceil$, which is a lower bound on $\operatorname{opt}_{k}(G, \mathcal{W})$. However, as indicated by the graph in Figure 2, not all $k$-extensions of $(G, \mathcal{W})$ have a complete admissible splitting at $s$.

In the sequel, we consider situations where a $k$-extension $H$ of $(G, \mathcal{W})$ has no admissible pair of edges. In [20], Z. Nutov gave some splitting off theorem under a more general setting in such a sense that it can be applied to any skew-supermodular set function $p$. However, we here show the splitting theorems and lemmas which are specified to $k$-NA-ECAP without applying Nutov's theorem, because we need to prove more than it for achieving a polynomial time algorithm to $k$-NA-ECAP, and because of the completeness of the paper. The following theorem is a key theorem for our algorithm.

Theorem 4.2 Let $(G=(V, E), \mathcal{W})$ be an area graph and $H=(V \cup\{s\}, E \cup F)$ be a k-extension of $(G, \mathcal{W})$ with $k \geq 2$ and an even $d_{H}(s)$. If no pair of two edge in $F$ is admissible, then we have $d_{H}(s)=4$ and $G$ has two components $C_{1}$ and $C_{2}$ with $d_{H}\left(s, C_{1}\right)=3$ and $d_{H}\left(s, C_{2}\right)=1$. Moreover, in the graph $H+e^{*}$ obtained by adding one arbitrary new edge $e^{*}$ to $E_{G}\left(C_{1}, C_{2}\right)$, there is a complete admissible splitting at $s$.

Before giving a proof of this theorem, we show several preparatory lemmas. For a graph $G=(V, E)$, every three cuts $X, Y$, and $Z$ satisfy the following inequality.

$$
\begin{aligned}
d_{G}(X)+d_{G}(Y)+d_{G}(Z) \geq & d_{G}(X-Y-Z)+d_{G}(Y-X-Z) \\
& +d_{G}(Z-X-Y)+d_{G}(X \cap Y \cap Z) \\
& +2 d_{G}(X \cap Y \cap Z, V-(X \cup Y \cup Z)) .
\end{aligned}
$$


For a $k$-extension $H=(V \cup\{s\}, E \cup F)$ of $(G, \mathcal{W})$, a pair $\{(s, u),(s, v)\} \subseteq F$ of two edges is not admissible if there is a cut $Y \subset V$ of type (A) or (B) with $\{u, v\} \subseteq Y$ and $d_{H}(Y) \leq k+1$. Such cut $Y$ is called a dangerous cut. Conversely, a pair $\{(s, u),(s, v)\}$ is not admissible only if there is a dangerous cut $Y \subset V$ with $\{u, v\} \subseteq Y$. We give the following two lemmas on the properties of dangerous cuts.

Lemma 4.3 Let $H=(V \cup\{s\}, E \cup F)$ be a k-extension of $(G=(V, E), \mathcal{W})$ and $Y \subset V$ be a dangerous cut. Then we have $d_{H}(s, V-Y) \geq d_{H}(s, Y)-1>0$.

Proof: Since $Y$ is a dangerous cut, we have $d_{H}(Y)=d_{H}(s, Y)+d_{H}(Y, V-Y) \leq k+1$. Moreover, $Y$ is of type (A) or (B), and hence so is $V-Y$, which implies $d_{H}(V-Y)=d_{H}(s, V-$ $Y)+d_{H}(Y, V-Y) \geq k$ by (4.1). Hence we have $d_{H}(s, V-Y) \geq k-d_{H}(Y, V-Y) \geq d_{H}(s, Y)-1$. From the definition of dangerous cuts, $d_{H}(s, Y) \geq 2$ holds.

Lemma 4.4 Let $H=(V \cup\{s\}, E \cup F)$ be a k-extension of $(G=(V, E), \mathcal{W})$ with an even $d_{H}(s)$. Assume that there are two dangerous cuts $Y_{1}, Y_{2} \subset V$ with $d_{H}\left(s, Y_{1}-Y_{2}\right)>0, d_{H}\left(s, Y_{2}-Y_{1}\right)>0$, and $d_{H}\left(s, Y_{1} \cap Y_{2}\right)>0$. Then we have $d_{H}\left(s, V-Y_{1}-Y_{2}\right)>0$.

Proof: Assume $d_{H}\left(s, Y_{1}-Y_{2}\right) \geq d_{H}\left(s, Y_{2}-Y_{1}\right)$ without loss of generality. By Lemma 4.3, we have $d_{H}\left(s, Y_{2}-Y_{1}\right)+d_{H}\left(s, V-Y_{1}-Y_{2}\right)=d_{H}\left(s, V-Y_{1}\right) \geq d_{H}\left(s, Y_{1}\right)-1=d_{H}\left(s, Y_{1}-Y_{2}\right)+$ $d_{H}\left(s, Y_{1} \cap Y_{2}\right)-1 \geq d_{H}\left(s, Y_{2}-Y_{1}\right)+d_{H}\left(s, Y_{1} \cap Y_{2}\right)-1$. Hence we have $d_{H}\left(s, V-Y_{1}-Y_{2}\right)=0$ would imply that the above inequalities hold by equality since $d_{H}\left(s, Y_{1} \cap Y_{2}\right) \geq 1$. This means $d_{H}\left(Y_{1}-Y_{2}\right)=d_{H}\left(Y_{2}-Y_{1}\right)$, which implies $d_{H}(s)=2 d_{H}\left(Y_{1}-Y_{2}\right)+1$, contradicting that $d_{H}(s)$ is even.

We show the following properties for cuts $Y$ of type (A) or (B) with $d_{H}(Y) \leq k+1$ (note that $Y$ is not necessarily dangerous).

Lemma 4.5 Let $H=(V \cup\{s\}, E \cup F)$ be a k-extension of $(G=(V, E), \mathcal{W})$ with $k \geq 2$. For every cut $Y \subset V$ of type $(A)$ with $d_{H}(Y) \leq k+1, \lambda(G[Y]) \geq k-\left\lfloor\frac{d_{H}(Y)}{2}\right\rfloor(\geq 1)$ holds.

Proof: By (4.2), for any partition $\left\{Y_{1}, Y_{2}\right\}$ of $Y, Y_{i}$ is also of type (A). Hence by (4.1), we have $d_{H}\left(Y_{i}\right) \geq k$ for $i=1,2$, and $d_{H}\left(Y_{1}, Y_{2}\right)=\left\lfloor\frac{1}{2}\left(d_{H}\left(Y_{1}\right)+d_{H}\left(Y_{2}\right)\right)\right\rfloor-\left\lfloor\frac{d_{H}(Y)}{2}\right\rfloor \geq k-\left\lfloor\frac{d_{H}(Y)}{2}\right\rfloor \geq 1$.

Lemma 4.6 Let $H=(V \cup\{s\}, E \cup F)$ be a k-extension of $(G=(V, E), \mathcal{W})$, and $Y_{1}$ and $Y_{2}$ be two cuts with $d_{H}\left(Y_{1}\right) \leq k+1, d_{H}\left(Y_{2}\right) \leq k+1$, and $d_{H}\left(Y_{1} \cap Y_{2},(V \cup\{s\})-\left(Y_{1} \cup Y_{2}\right)\right)>0$ such that both $Y_{1}$ and $Y_{2}$ are of type $(A)$ or of type $(B)$. If $Y_{1}$ and $Y_{2}$ cross each other in $H$, then both of the cuts $Y_{1}-Y_{2}$ and $Y_{2}-Y_{1}$ are of type $(A)$ and we have $d_{H}\left(Y_{1}\right)=d_{H}\left(Y_{2}\right)=k+1$, $d_{H}\left(Y_{1}-Y_{2}\right)=d_{H}\left(Y_{2}-Y_{1}\right)=k$, and $d_{H}\left(Y_{1} \cap Y_{2},(V \cup\{s\})-\left(Y_{1} \cup Y_{2}\right)\right)=1$. 

and $Y_{2}-Y_{1}$ are of type $(A)$ by (4.2) (resp., by $\left(Y_{1}-Y_{2}\right) \cap W_{2}=\emptyset=\left(Y_{2}-Y_{1}\right) \cap W_{1}$, where $W_{i}$ denotes an area with $\left.W_{i} \subseteq Y_{i}\right)$. Hence, $d_{H}\left(Y_{1}-Y_{2}\right) \geq k$ and $d_{H}\left(Y_{2}-Y_{1}\right) \geq k$ hold by (4.1). By $d_{H}\left(Y_{1} \cap Y_{2},(V \cup\{s\})-\left(Y_{1} \cup Y_{2}\right)\right)>0, d_{H}\left(Y_{1}-Y_{2}\right) \geq k, d_{H}\left(Y_{2}-Y_{1}\right) \geq k$, and (2.1), we have $2(k+1) \geq d_{H}\left(Y_{1}\right)+d_{H}\left(Y_{2}\right)=d_{H}\left(Y_{1}-Y_{2}\right)+d_{H}\left(Y_{2}-Y_{1}\right)+2 d_{H}\left(Y_{1} \cap Y_{2},(V \cup\{s\})-\left(Y_{1} \cup Y_{2}\right)\right)$ $\geq k+k+2$. This proves the lemma.

We first show Theorem 4.2 in a special case where $d_{H}(s, C) \leq 1$ holds for every component $C$ in $G$. In this case, we can see that there is a complete admissible splitting at $s$.

Lemma 4.7 Let $G, H$, and $k$ satisfy the assumption of Theorem 4.2. If $d_{H}(s, C) \leq 1$ holds for each component in $G$, then there is a complete admissible splitting at $s$.

Proof: We prove the lemma by showing that every pair of two edges in $F$ is admissible. Let $\mathcal{C}=\left\{C_{1}, \ldots, C_{q}\right\}$ denote the family of all components with $d_{H}\left(s, C_{i}\right)=1, C_{i} \subseteq V$, where $\left\{\left(s, v_{i}\right)\right\}=E_{H}\left(s, C_{i}\right)$. Then every area $W \in \mathcal{W}$ satisfies $W \cap C_{i} \neq \emptyset$ for any $C_{i} \in \mathcal{C}$ since a component $C \in \mathcal{C}$ with $W \cap C=\emptyset$ is of type (A) and satisfies $d_{H}\left(C_{i}\right)=d_{H}\left(s, C_{i}\right) \geq k$ by (4.1), contradicting $k \geq 2$.

Assume by contradiction that some pair $\left\{\left(s, v_{1}\right),\left(s, v_{2}\right)\right\}$ is not admissible. Then there is a dangerous cut $Y \subset V$ with $\left\{v_{1}, v_{2}\right\} \subseteq Y$. Lemma 4.5 implies that $Y$ is of type (B) since two vertices $v_{1}$ and $v_{2}$ are contained in distinct components in $G$ and hence $G[Y]$ is not connected. Let $W_{1} \in \mathcal{W}$ be an area with $W_{1} \subseteq Y$. Lemma 4.3 says that there is an edge $\left(s, v_{3}\right) \in$ $E_{H}(s, V-Y)$. By $\left(C_{3}-Y\right) \cap W_{1}=\emptyset$ and (4.1), we have $d_{H}\left(C_{3}-Y\right) \geq k$. Hence $d_{G}\left(C_{3}-Y\right)=$ $d_{G}\left(C_{3}-Y, C_{3} \cap Y\right) \geq k-1$ by $C_{3} \cap W_{1} \neq \emptyset$ and $\left(s, v_{3}\right) \in E_{H}(s, V-Y)$. Hence we have $d_{H}(Y) \geq d_{H}(s, Y)+d_{G}\left(C_{3}-Y, C_{3} \cap Y\right) \geq k+1$ by $d_{H}(s, Y) \geq 2$. By $d_{H}(Y) \leq k+1$, we have $d_{H}(Y)=k+1, d_{H}(s, Y)=2$, and $d_{G}(Y)=d_{G}\left(C_{3}-Y, C_{3} \cap Y\right)$. This means that the number of components $C \in \mathcal{C}$ with $C \subseteq Y$ is two and the number of components $C \in \mathcal{C}$ with $C-Y \neq \emptyset$ is one. There is no other edge $\left(s, v_{j}\right) \in E_{H}(s, V-Y), j \neq 3$ because if $C_{j}-Y \neq \emptyset$ holds for another component $C_{j} \in \mathcal{C}$, then $d_{G}\left(C_{j} \cap Y, C_{j}-Y\right)>0$ holds by $C_{j} \cap W_{1} \neq \emptyset$, from which $d_{H}(Y)>k+1$ would hold. Hence $|\mathcal{C}|=3$ holds. It contradicts that $d_{H}(s)$ is even.

The following theorem shows a property of a $k$-extension of $(G, \mathcal{W})$ which has no edge incident to $s$ admissible with a fixed edge $(s, u)$.

Theorem 4.8 Let $H=(V \cup\{s\}, E \cup F)$ be a k-extension of $(G=(V, E), \mathcal{W})$ with an even $d_{H}(s)$ and $k \geq 2$, and $(s, u) \in F$ be an edge. If $\{(s, u), e\}$ is not admissible for any edge $e \in F$, then there are three dangerous cuts $Y_{1}, Y_{2}$, and $Y_{3}$ with $u \in Y_{1} \cap Y_{2} \cap Y_{3}, d_{H}\left(Y_{1}\right)=d_{H}\left(Y_{2}\right)=$ $d_{H}\left(Y_{3}\right)=k+1, d_{H}\left(s, Y_{1}-Y_{2}-Y_{3}\right)>0, d_{H}\left(s, Y_{2}-Y_{1}-Y_{3}\right)>0$, and $d_{H}\left(s, Y_{3}-Y_{1}-Y_{2}\right)>0$ such that one of the following $(i)$ and (ii) holds. 
(i) Each $Y_{i}$ is of type $(B)$. We have $d_{G}\left(Y_{1} \cap Y_{2} \cap Y_{3}\right)=0, d_{H}\left(s, Y_{1} \cap Y_{2} \cap Y_{3}\right)=1$, and $d_{H}\left(Y_{1}-Y_{2}-Y_{3}\right)=d_{H}\left(Y_{2}-Y_{1}-Y_{3}\right)=d_{H}\left(Y_{3}-Y_{1}-Y_{2}\right)=k$.

(ii) Both of $Y_{1}$ and $Y_{2}$ are of type $(A)$ and $Y_{3}$ is of type $(B)$ (without loss of generality). We have $d_{G}\left(Y_{3}-Y_{1}-Y_{2}\right)=0, d_{H}\left(s, Y_{3}-Y_{1}-Y_{2}\right)=1$, and $d_{H}\left(Y_{1}-Y_{2}-Y_{3}\right)=d_{H}\left(Y_{2}-Y_{1}-Y_{3}\right)=$ $d_{H}\left(Y_{1} \cap Y_{2} \cap Y_{3}\right)=k$. Moreover, $V=Y_{1} \cup Y_{2} \cup Y_{3}$ holds.

Proof: Since $\left\{(s, u),\left(s, v_{i}\right)\right\}$ is non-admissible for any edge $\left(s, v_{i}\right) \in F-\{(s, u)\}$, there is a dangerous cut containing $u$ and $v_{i}$ in $H$. For each edge $\left(s, v_{i}\right) \in F-\{(s, u)\}$, let $Y_{i}$ denote a dangerous cut containing two vertices $u$ and $v_{i}$. Let $\mathcal{Y}=\left\{Y_{i} \mid\left(s, v_{i}\right) \in F-\{(s, u)\}\right\}$. Lemma 4.3 implies $|\mathcal{Y}| \geq 2$. We first claim that for any two cuts $Y_{1}, Y_{2}$ in $\mathcal{Y}$,

$$
d_{H}\left(s, V-Y_{1}-Y_{2}\right)>0 \text { holds. }
$$

By Lemma 4.4 and $d_{H}\left(s, Y_{1} \cap Y_{2}\right)>0$, (4.5) holds if $d_{H}\left(s, Y_{1}-Y_{2}\right)>0$ and $d_{H}\left(s, Y_{2}-Y_{1}\right)>0$ hold. On the other hand, assume $d_{H}\left(s, Y_{1}-Y_{2}\right)=0$ without loss of generality. Then we have $d_{H}\left(s, Y_{1} \cup Y_{2}\right)=d_{H}\left(Y_{2}\right)$. By Lemma 4.3 and $E_{H}\left(s, Y_{2}\right) \supseteq E_{H}\left(s, Y_{1}\right)$, we have $d_{H}\left(s, V-Y_{1}-Y_{2}\right)>$ 0 .

By (4.5), there are an edge $\left(s, v_{3}\right) \in E_{H}\left(s, V-Y_{1}-Y_{2}\right)$ and the corresponding dangerous cut $Y_{3} \in \mathcal{Y}$ with $\left\{u, v_{3}\right\} \subseteq Y_{3}$. Then we can see the following claim.

Claim 4.9 For three cuts $Y_{1}, Y_{2}, Y_{3} \in \mathcal{Y}$ such that $d_{H}\left(s, Y_{3}-Y_{1}-Y_{2}\right)>0$ and $d_{H}\left(s, Y_{1} \cup Y_{2}\right) \geq$ $\max \left\{d_{H}\left(s, Y_{1} \cup Y_{3}\right), d_{H}\left(s, Y_{2} \cup Y_{3}\right)\right\}$, we have

$$
d_{H}\left(s, Y_{1}-Y_{2}-Y_{3}\right)>0, d_{H}\left(s, Y_{2}-Y_{1}-Y_{3}\right)>0, \text { and } d_{H}\left(s, Y_{3}-Y_{1}-Y_{2}\right)>0 .
$$

Hence by choosing $Y_{1}, Y_{2} \in \mathcal{Y}$ such that $d_{H}\left(s, Y_{1} \cup Y_{2}\right)$ is the maximum, $Y_{1}, Y_{2}$, and $Y_{3}$ satisfy (4.6).

Then there are the following four possible cases.

(Case-1) Each cut $Y_{i}, i=1,2,3$ is of type (A).

(Case-2) Each cut $Y_{i}, i=1,2,3$ is of type (B).

(Case-3) $Y_{1}$ is of type (A) and both of $Y_{2}$ and $Y_{3}$ are of type (B).

(Case-4) Both of $Y_{1}$ and $Y_{2}$ are of type (A) and $Y_{3}$ is of type (B).

Let $W_{i}$ denote an area in $\mathcal{W}$ with $W_{i} \subseteq Y_{i}$ if $Y_{i}$ is of type (B). Note that in each case, every $Y_{i}$ satisfies $d_{H}\left(Y_{i}\right) \leq k+1$ for $i=1,2,3$, and $d_{H}\left(Y_{1} \cap Y_{2} \cap Y_{3}, V \cup\{s\}-\left(Y_{1} \cup Y_{2} \cup Y_{3}\right)\right) \geq$ $d_{H}\left(s, Y_{1} \cap Y_{2} \cap Y_{3}\right) \geq d_{H}(s, u)>0$ holds. By (4.6), we have $Y_{1}-Y_{2}-Y_{3} \neq \emptyset, Y_{2}-Y_{3}-Y_{1} \neq \emptyset$, and $Y_{3}-Y_{1}-Y_{2} \neq \emptyset$.

(Case-1) By (4.1) and (4.2), we have $d_{H}\left(Y_{1}-Y_{2}-Y_{3}\right) \geq k, d_{H}\left(Y_{2}-Y_{3}-Y_{1}\right) \geq k, d_{H}\left(Y_{3}-Y_{1}-\right.$ $\left.Y_{2}\right) \geq k$, and $d_{H}\left(Y_{1} \cap Y_{2} \cap Y_{3}\right) \geq k$. By (4.4), we have $3(k+1) \geq \sum_{i=1}^{3} d_{H}\left(Y_{i}\right) \geq d_{H}\left(Y_{1}-Y_{2}-Y_{3}\right)+$ 
$d_{H}\left(Y_{2}-Y_{3}-Y_{1}\right)+d_{H}\left(Y_{3}-Y_{1}-Y_{2}\right)+d_{H}\left(Y_{1} \cap Y_{2} \cap Y_{3}\right)+2 d_{H}\left(Y_{1} \cap Y_{2} \cap Y_{3}, V \cup\{s\}-\left(Y_{1} \cup Y_{2} \cup Y_{3}\right)\right)$ $\geq 4 k+2$, contradicting $k \geq 2$. This case cannot occur.

(Case-2) By $\left(Y_{1}-Y_{2}-Y_{3}\right) \cap W_{2}=\emptyset$, the cut $Y_{1}-Y_{2}-Y_{3}$ is of type (A) and hence satisfies $d_{H}\left(Y_{1}-Y_{2}-Y_{3}\right) \geq k$ by (4.1). Similarly, we have $d_{H}\left(Y_{2}-Y_{3}-Y_{1}\right) \geq k$ and $d_{H}\left(Y_{3}-Y_{1}-Y_{2}\right) \geq k$. We have $d_{H}\left(Y_{1} \cap Y_{2} \cap Y_{3}\right) \geq d_{H}\left(s, Y_{1} \cap Y_{2} \cap Y_{3}\right) \geq 1$. By (4.4), we have $3(k+1) \geq \sum_{i=1}^{3} d_{H}\left(Y_{i}\right) \geq$ $d_{H}\left(Y_{1}-Y_{2}-Y_{3}\right)+d_{H}\left(Y_{2}-Y_{3}-Y_{1}\right)+d_{H}\left(Y_{3}-Y_{1}-Y_{2}\right)+d_{H}\left(Y_{1} \cap Y_{2} \cap Y_{3}\right)+2 d_{H}\left(Y_{1} \cap Y_{2} \cap\right.$ $\left.Y_{3}, V \cup\{s\}-\left(Y_{1} \cup Y_{2} \cup Y_{3}\right)\right) \geq 3 k+3$. This implies that every inequality turns out to be an equality. The cuts $Y_{1}, Y_{2}$, and $Y_{3}$ satisfy the statement (i) of this theorem.

(Case-3) By (4.1) and (4.2), we have $d_{H}\left(Y_{1}-Y_{2}-Y_{3}\right) \geq k$ and $d_{H}\left(Y_{1} \cap Y_{2} \cap Y_{3}\right) \geq k$. By $\left(Y_{2}-Y_{3}-Y_{1}\right) \cap W_{3}=\emptyset$ and (4.1), we have $d_{H}\left(Y_{2}-Y_{3}-Y_{1}\right) \geq k$. Similarly, $d_{H}\left(Y_{3}-Y_{1}-Y_{2}\right) \geq k$ holds. Similarly to Case-1, by $k \geq 2$ and (4.4), we see that this case cannot occur.

(Case-4) By (4.1) and (4.2), we have $d_{H}\left(Y_{1}-Y_{2}-Y_{3}\right) \geq k, d_{H}\left(Y_{2}-Y_{3}-Y_{1}\right) \geq k$, and $d_{H}\left(Y_{1} \cap Y_{2} \cap Y_{3}\right) \geq k$. By (4.6), we have $d_{H}\left(Y_{3}-Y_{1}-Y_{2}\right) \geq d_{H}\left(s, Y_{3}-Y_{1}-Y_{2}\right) \geq 1$. Similarly to Case-2, we see by (4.4) that every inequality turns out to be an equality. Hence the cuts $Y_{1}$, $Y_{2}$, and $Y_{3}$ satisfy the statement (ii) of this theorem, except $V=Y_{1} \cup Y_{2} \cup Y_{3}$.

Let $Y_{1}, Y_{2}, Y_{3}$ be three cuts satisfying Case- 4 such that $d_{H}\left(s, Y_{1} \cup Y_{2} \cup Y_{3}\right)$ is the maximum, and $W_{3}$ be an area in $\mathcal{W}$ with $W_{3} \subseteq Y_{3} \cdot d_{H}\left(s, Y_{3}-Y_{1}-Y_{2}\right)=1$ means that $d_{H}\left(s, Y_{1} \cup Y_{2}\right)$ is the maximum. We now have $d_{G}\left(Y_{3}-Y_{1}-Y_{2}\right)=0$.

Assume by contradiction that $V-Y_{1}-Y_{2}-Y_{3} \neq \emptyset$ holds. We show the following claim.

Claim 4.10 $E_{H}\left(s, V-\left(Y_{1} \cup Y_{2} \cup Y_{3}\right)\right)=\emptyset$ holds.

Proof: Assume by contradiction that there is an edge $\left(s, v_{4}\right) \in E_{H}\left(s, V-\left(Y_{1} \cup Y_{2} \cup Y_{3}\right)\right)$. Let $Y_{4}$ be the corresponding dangerous cut in $\mathcal{Y}$ with $\left\{u, v_{4}\right\} \subseteq Y_{4}$. Assume that $Y_{4}$ is of type (A). Then by $d_{G}\left(Y_{3}-Y_{1}-Y_{2}\right)=0$ and Lemma 4.5 , we have $Y_{4} \cap\left(Y_{3}-Y_{1}-Y_{2}\right)=\emptyset$. Hence from the maximality of $d_{H}\left(s, Y_{1} \cup Y_{2}\right)$, the cuts $Y_{1}, Y_{2}$, and $Y_{4}$ satisfy Case-1, which cannot occur. Assume that $Y_{4}$ is of type (B) and the cuts $Y_{1}, Y_{2}$, and $Y_{4}$ satisfy Case-4. Then we have $d_{G}\left(Y_{4}-Y_{1}-Y_{2}\right)=0$ and $d_{H}\left(Y_{4}-Y_{1}-Y_{2}\right)=1$, implying that $Z=Y_{4}-Y_{1}-Y_{2}-\left(Y_{3}-Y_{1}-Y_{2}\right)$ satisfies $Z \cap W_{3}=\emptyset$ but $d_{H}(Z)=1$, a contradiction to (4.1). Assume that $Y_{4}$ is of type (B) and the cuts $Y_{1}, Y_{2}$, and $Y_{4}$ do not satisfy Case-4. Then we have $d_{H}\left(s, Y_{1}-Y_{2}-Y_{4}\right)=0$ without loss of generality. The cuts $Y_{2}, Y_{3}$, and $Y_{4}$ do not satisfy Case- 3 from the above arguments, and hence $d_{H}\left(s, Y_{3}-Y_{2}-Y_{4}\right)=0$ or $d_{H}\left(s, Y_{2}-Y_{3}-Y_{4}\right)=0$ hold. This means $d_{H}\left(s, Y_{2} \cup Y_{4}\right)>$ $d_{H}\left(s, Y_{1} \cup Y_{2} \cup Y_{3}\right)$ or $d_{H}\left(s, Y_{3} \cup Y_{4}\right)>d_{H}\left(s, Y_{1} \cup Y_{2} \cup Y_{3}\right)$. This and Lemma 4.4 imply that there are three dangerous cuts $Y_{1}^{\prime}, Y_{2}^{\prime}$, and $Y_{3}^{\prime}$ satisfying Case-4 with $d_{H}\left(s, Y_{1}^{\prime} \cup Y_{2}^{\prime} \cup Y_{3}^{\prime}\right)>$ $d_{H}\left(s, Y_{1} \cup Y_{2} \cup Y_{3}\right)$, contradicting the maximality of $d_{H}\left(s, Y_{1} \cup Y_{2} \cup Y_{3}\right)$ (note that such cuts $Y_{1}^{\prime}, Y_{2}^{\prime}$, and $Y_{3}^{\prime}$ do not satisfy Case-2 since otherwise the component $C^{\prime}$ of $G$ with $u \in C^{\prime}$ satisfies $d_{H}\left(s, C^{\prime}\right)=d_{H}(s, u)=1$, contradicting that the cut $Y_{1}$ satisfies $u \in Y_{1}$ and $d_{H}\left(s, Y_{1}\right) \geq 2$ and $G\left[Y_{1}\right]$ is connected by Lemma 4.5). Therefore we have $E_{H}\left(s, V-\left(Y_{1} \cup Y_{2} \cup Y_{3}\right)\right)=\emptyset$. 
Proof of Theorem 4.2: Assume that $H$ has no admissible pair of two edges in $F$. Let $C_{1}$ denote a component in $G$ with $d_{H}\left(s, C_{1}\right) \geq 2$ and $\left(s, u_{0}\right) \in E_{H}\left(s, C_{1}\right)$. Then Theorem 4.8 says that there are three dangerous cuts $Y_{1}, Y_{2}$, and $Y_{3}$ satisfying (i) or (ii) in Theorem 4.8 for $u=u_{0}$. If they satisfy (i), then $d_{H}\left(s, Y_{1} \cap Y_{2} \cap Y_{3}\right)=d_{H}\left(s, u_{0}\right)=1$ and $d_{G}\left(Y_{1} \cap Y_{2} \cap Y_{3}\right)=0$ hold, which implies that $C_{1} \subseteq Y_{1} \cap Y_{2} \cap Y_{3}$ holds, contradicting $d_{H}\left(s, C_{1}\right) \geq 2$. Hence such three dangerous cuts $Y_{1}, Y_{2}$, and $Y_{3}$ satisfy (ii) in Theorem 4.8. Let $Y_{1}, Y_{2}$ be of type (A) and $Y_{3}$ be of type (B). Since Lemma 4.5 says that both of $G\left[Y_{1}\right]$ and $G\left[Y_{2}\right]$ are connected, $G\left[Y_{1} \cup Y_{2}\right]$ is also connected. By Theorem 4.8, $d_{H}\left(s, Y_{3}-Y_{1}-Y_{2}\right)=1$ and $d_{G}\left(Y_{3}-Y_{1}-Y_{2}\right)=0$ hold. Hence we have $C_{1}=Y_{1} \cup Y_{2}$. Let $\left(s, u_{3}\right)$ be the edge with $\left\{\left(s, u_{3}\right)\right\}=E_{H}\left(s, Y_{3}-Y_{1}-Y_{2}\right)$ and $C_{2}$ be the component in $G$ with $u_{3} \in C_{2}$.

We show that $d_{H}(s)=4$ holds. Now $Y_{1}$ and $Y_{2}$ cross each other in $H$. Lemma 4.6 says that

$$
d_{H}\left(Y_{1}-Y_{2}\right)=d_{H}\left(Y_{2}-Y_{1}\right)=k,
$$

$d_{H}\left(s, Y_{1} \cap Y_{2}\right)=1$, and $E_{H}\left(s, Y_{1} \cap Y_{2}\right)=\left\{\left(s, u_{0}\right)\right\}$ hold. Hence it suffices to show that $d_{H}\left(s, Y_{1}-\right.$ $\left.Y_{2}\right)=d_{H}\left(s, Y_{2}-Y_{1}\right)=1$ holds.

We here show that $d_{H}\left(s, Y_{1}-Y_{2}\right)=1$ holds $\left(d_{H}\left(s, Y_{2}-Y_{1}\right)=1\right.$ can be proved similarly). Assume by contradiction that $d_{H}\left(s, Y_{1}-Y_{2}\right) \geq 2$ holds. For an edge $\left(s, u_{1}\right) \in E_{H}\left(s, Y_{1}-Y_{2}\right)$, no pair $\left\{\left(s, u_{1}\right), e\right\}$ with $e \in F-\left\{\left(s, u_{1}\right)\right\}$ is admissible in $H$. By Theorem 4.8, $H$ has three dangerous cuts $Z_{1}, Z_{2}$, and $Z_{3}$ with $u_{1} \in Z_{1} \cap Z_{2} \cap Z_{3}$ and satisfying (i) or (ii) in Theorem 4.8 for $u=u_{1}$. Since $d_{H}\left(s, C_{1}\right) \geq 2$ holds, the cuts $Z_{1}, Z_{2}$, and $Z_{3}$ satisfy (ii). Let $Z_{1}, Z_{2}$ be of type (A) and $Z_{3}$ be of type (B). We have

$$
V=Z_{1} \cup Z_{2} \cup Z_{3}, d_{H}\left(s, Z_{3}-Z_{1}-Z_{2}\right)=1
$$

Then two cuts $Z_{1}$ and $Y_{1}-Y_{2}$ do not cross each other in $H$, since otherwise Lemma 4.6 says $d_{H}\left(Y_{1}-Y_{2}\right)=k+1$, contradicting (4.7) (note that both of $Z_{1}$ and $Y_{1}-Y_{2}$ are of type (A) and we have $d_{H}\left(Z_{1}\right) \leq k+1, d_{H}\left(Y_{1}-Y_{2}\right)=k$, and $\left.\left(s, u_{1}\right) \in E_{H}\left(s,\left(Y_{1}-Y_{2}\right) \cap Z_{1}\right)\right)$. Similarly, two cuts $Z_{2}$ and $Y_{1}-Y_{2}$ do not cross each other in $H$. By these properties, $d_{H}\left(s, Z_{1}-Z_{2}\right)>0$, and $d_{H}\left(s, Z_{2}-Z_{1}\right)>0$, there are two possible cases of (a) $Y_{1}-Y_{2} \subseteq Z_{1} \cap Z_{2}$, and (b) $Y_{1}-Y_{2} \supseteq Z_{1} \cup Z_{2}$. In the case of (a), by applying Lemma 4.6 to $Z_{1}$ and $Z_{2}$, we have $d_{H}\left(s, Z_{1} \cap Z_{2}\right)=1$, contradicting 

From this and $d_{H}\left(s, Y_{2}\right) \geq 2, d_{H}\left(s, Z_{3}-Z_{1}-Z_{2}\right) \geq 2$ holds, contradicting (4.8).

We finally show that after adding one edge $e^{*}$ to $E_{H}\left(C_{1}, C_{2}\right)$, there is a complete splitting at $s$ while preserving (4.1) in $H+e^{*}$. In $H^{\prime}=H+e^{*}$, all neighbors of $s$ are contained in the single component $C_{1} \cup C_{2}$ in $H^{\prime}[V]$. This implies that for any edge $(s, u) \in F$, there is another edge $(s, v) \in F-\{(s, u)\}$ such that $\{(s, u),(s, v)\}$ is admissible, since otherwise Theorem 4.8 (i) or (ii) hold, that is, there is a component $C^{\prime}$ in $H^{\prime}[V]$ with $d_{H}\left(s, C^{\prime}\right)=1$, contradicting that all neighbors of $s$ in $H^{\prime}$ are contained in the single component in $H^{\prime}[V]$. Since all neighbors of $s$ remain to be contained in the component $C_{1} \cup C_{2}$ after any splitting at $s$, we see that there is a complete splitting at $s$ while preserving (4.1) in $H+e^{*}$.

Before closing this section, we show that if at least one area is included in a component, then there is a complete admissible splitting at $s$ in $H$, and, $\operatorname{opt}_{k}(G, \mathcal{W})=\left\lceil\alpha_{k}(G, \mathcal{W}) / 2\right\rceil$ holds.

Theorem 4.11 For the $k$-NA-ECAP with $k \geq 2$, opt $t_{k}(G, \mathcal{W})=\left\lceil\alpha_{k}(G, \mathcal{W}) / 2\right\rceil$ holds if at least one area is included in a component of $G$.

Proof: Let $H=(V \cup\{s\}, E \cup F)$ be a critical $k$-extension of $(G, \mathcal{W})$. If $|F|$ is odd, then we add one extra edge conncecting $s$ and a vertex in $V$ to $F$ and redenote the resulting graph by $H ;|F| / 2=\left\lceil\alpha_{k}(G) / 2\right\rceil$ holds by Theorem 4.1. It suffices to show that in $H$, there is an edge $e^{\prime} \in F-\{e\}$ such that $\left\{e, e^{\prime}\right\}$ is admissible in $H$ for any edge $e \in F$. Let $\mathcal{C}$ be the family of all components in $G$. Since some area $W \in \mathcal{W}$ satisfies $W \subseteq C$, then each $C \in \mathcal{C}$ is of type (A) or (B) and hence satisfies $d_{H}(C)=d_{H}(s, C) \geq k \geq 2$ by (4.1) and $k \geq 2$. Hence for any edge $e \in F$, there is an edge $e^{\prime} \in F-\{e\}$ such that $\left\{e, e^{\prime}\right\}$ is admissible in $H$, since otherwise Theorem 4.8 says that some $C^{\prime} \in \mathcal{C}$ satisfies $d_{H}\left(s, C^{\prime}\right)=1$, a contradiction.

\section{$5 \quad$ Algorithm}

Based on the lower bounds in Section 3 and theorems about extensions and splittings in Section 4, we give an algorithm, called NAEC-AUG, for finding a feasible solution $E^{\prime}$ to $k$-NA-ECAP with $\left|E^{\prime}\right|=\operatorname{opt}_{k}(G, \mathcal{W})$, for a given area graph $(G, \mathcal{W})$ and an integer $k \geq 3$. It finds a feasible solution $E^{\prime}$ with $\left|E^{\prime}\right|=\left\lceil\alpha_{k}(G, \mathcal{W}) / 2\right\rceil+1$ if $(G, \mathcal{W})$ has property $(\mathrm{P}),\left|E^{\prime}\right|=\left\lceil\alpha_{k}(G, \mathcal{W}) / 2\right\rceil$ otherwise. The algorithm is based on edge-splitting operations and may resplit some edges which have been split off. For a graph $H=(V \cup\{s\}, E)$ and a designated vertex $s \notin V$, we say that $H^{\prime}$ is obtained from $H$ by hooking up an edge $(u, v) \in E(H-s)$ at $s$, if we construct $H^{\prime}$ by replacing the edge $(u, v)$ with two edges $(s, u)$ and $(s, v)$ in $H$.

The outline of algorithm NAEC-AUG is described as follows. In the first step, we first obtain a critical $k$-extension $H=\left(V \cup\{s\}, E \cup F_{1}\right)$ of a given $(G, \mathcal{W})$. Theorem 4.1 says that 


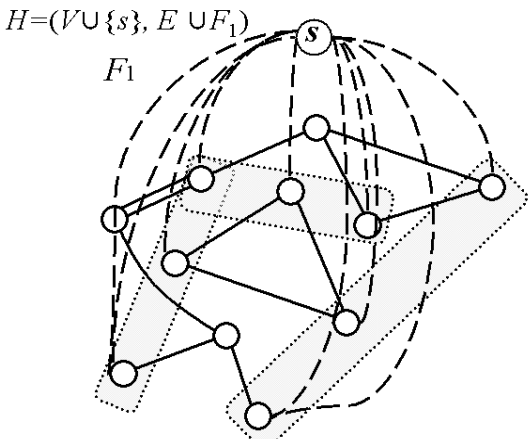

(i)

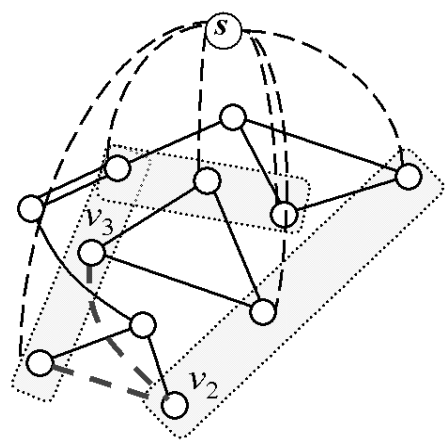

(iii)

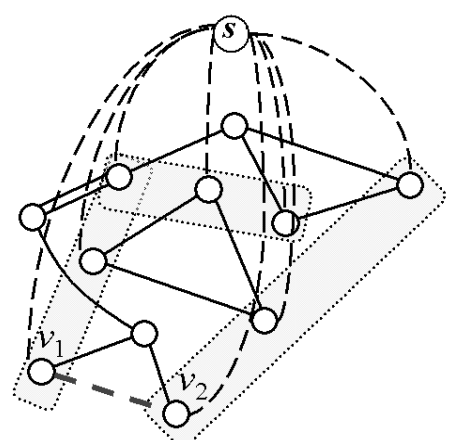

(ii)

(S)

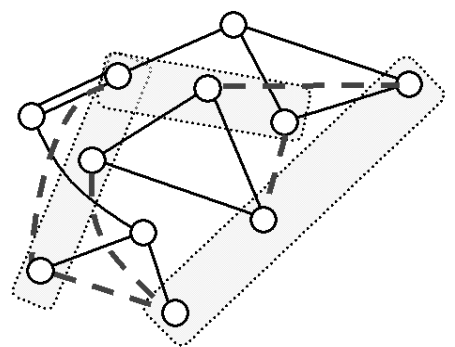

(iv)

Figure 3: Computational process of algorithm NAEC-AUG applied to $k=3$ and the area graph $(G, \mathcal{W})$ in Figure 1. The lower bound in Section 3 is $\left\lceil\alpha_{3}(G, \mathcal{W}) / 2\right\rceil=5$. (i) A critical $k$-extension $H=\left(V \cup\{s\}, E \cup F_{1}\right)$ of $(G, \mathcal{W})$ obtained by Step 1. Edges in $F_{1}$ are drawn as broken lines. Then $\lambda_{H}(v, W) \geq 3$ holds for every pair of $v \in V$ and $W \in \mathcal{W}$. (ii) $H_{1}=$ $\left(H-\left\{\left(s, v_{1}\right),\left(s, v_{2}\right)\right\}\right) \cup\left\{\left(v_{1}, v_{2}\right)\right\}$ obtained from $H$ by an admissible splitting of $\left(s, v_{1}\right)$ and $\left(s, v_{2}\right)$. (iii) $H_{2}=\left(H_{1}-\left\{\left(s, v_{2}\right),\left(s, v_{3}\right)\right\}\right) \cup\left\{\left(v_{2}, v_{3}\right)\right\}$ obtained from $H_{1}$ by the admissible splitting of $\left(s, v_{2}\right)$ and $\left(s, v_{3}\right)$. (iv) $H_{3}$ obtained from $H_{2}$ by a complete admissible splitting at $s$. The graph $G_{3}=H_{3}-s$ is 3 -NA-edge-connected.

$\left|F_{1}\right|=\alpha_{k}(G, \mathcal{W})$. If $\left|F_{1}\right|$ is odd, then we add an arbitrary one edge connecting $s$ and a vertex in $V$ to $F_{1}$. In the next step, we repeat admissible edge-splittings at $s$. If $(G, \mathcal{W})$ does not have property $(\mathrm{P})$, then the algorithm finds a complete admissible splitting, and hence the set $E^{*}$ of added edges satisfies $\left|E^{*}\right|=\left\lceil\alpha_{k}(G, \mathcal{W}) / 2\right\rceil$ and $\lambda\left(G+E^{*}, \mathcal{W}\right) \geq k$. If $(G, \mathcal{W})$ has property $(\mathrm{P})$, then the algorithm finds such a complete splitting by adding one extra edge to $G$, and hence the obtained edge set $E^{*}$ satisfies $\left|E^{*}\right|=\left\lceil\alpha_{k}(G, \mathcal{W}) / 2\right\rceil+1$. In both cases, $E^{*}$ is optimal by Lemmas 3.2 and 3.4 .

More precisely, we describe the algorithm below, and introduce one theorem necessary to justify the algorithm, which will be proved in Section 6. An example of computational process of NAEC-AUG is shown in Figure 3. 


\section{Algorithm NAEC-AUG}

Input: An area graph $(G=(V, E), \mathcal{W})$ and an integer $k \geq 3$.

Output: A set $E^{*}$ of new edges with $\lambda\left(G+E^{*}, \mathcal{W}\right) \geq k$ and $\left|E^{*}\right|=\operatorname{opt}_{k}(G, \mathcal{W})$.

Step 1: We find a critical $k$-extension $H=\left(V \cup\{s\}, E \cup F_{1}\right)$ of $(G, \mathcal{W})$. If $d_{H}(s)$ is odd, then we add to $F_{1}$ one extra edge between $s$ and $V$.

Step 2: We continue to execute admissible edge-splittings at $s$ until no pair of two edges incident to $s$ is admissible. Let $H_{2}=\left(V \cup\{s\}, E \cup E_{2} \cup F_{2}\right)$ be the resulting graph, where $F_{2}=E_{H_{2}}(s)$ and $E_{2}$ denotes the set of split edges.

If $F_{2}=\emptyset$ holds, then halt after outputting $E^{*}:=E_{2}$.

Otherwise $d_{H_{2}}(s)=4$ holds and the graph $H_{2}-s$ has two components $C_{1}$ and $C_{2}$ with $d_{H_{2}}\left(s, C_{1}\right)=3$ and $d_{H_{2}}\left(s, C_{2}\right)=1$, where $E_{H_{2}}\left(s, C_{2}\right)=\left\{\left(s, u^{*}\right)\right\}$ (they exist by Theorem 4.2). We have the following four cases $(\mathrm{a})-(\mathrm{d})$.

(a) The vertex $u^{*}$ is contained in no cut $X \subseteq C_{2}$ of type (A) with $d_{H_{2}}(X)=k$. Then after replacing $\left(s, u^{*}\right)$ with a new edge $(s, v)$ for some vertex $v \in C_{1}$ while preserving (4.1), execute a complete admissible splitting at $s$. Output the set $E^{*}$ of all split edges, where $\left|E^{*}\right|=\left\lceil\alpha_{k}(G, \mathcal{W}) / 2\right\rceil$ holds.

(b) $E_{2} \cap E\left(H_{2}\left[V-C_{1}\right]\right) \neq \emptyset$ holds. Then after hooking up one edge $e \in E_{2} \cap E\left(H_{2}\left[V-C_{1}\right]\right)$, execute a complete admissible splitting at $s$. Output the set $E^{*}$ of all split edges, where $\left|E^{*}\right|=$ $\left\lceil\alpha_{k}(G, \mathcal{W}) / 2\right\rceil$ holds.

(c) There is a set $E^{\prime} \subseteq E_{2}$ of at most two split edges such that the graph $H_{3}$ resulting from hooking up the set $E^{\prime}$ of edges in $H_{2}$ has an admissible pair $\left\{\left(s, u^{*}\right), f\right\}$ for some $f \in E_{H_{3}}(s, V)$. After a complete admissible splitting at $s$ in $H_{3}$, output the set $E^{*}$ of all split edges, where $\left|E^{*}\right|=\left\lceil\alpha_{k}(G, \mathcal{W}) / 2\right\rceil$ holds.

(d) None of $(\mathrm{a})-(\mathrm{c})$ holds. Then we can prove that $(G, \mathcal{W})$ has property $(\mathrm{P})$. After adding one new edge $e^{*}$ to $E_{H_{2}}\left(C_{1}, C_{2}\right)$, execute a complete admissible splitting at $s$ in $H_{2}+\left\{e^{*}\right\}$. Outputting the edge set $E^{*}:=E_{3} \cup\left\{e^{*}\right\}$, where $E_{3}$ denotes the set of all split edges and $\left|E^{*}\right|=\left\lceil\alpha_{k}(G, \mathcal{W}) / 2\right\rceil+1$ holds.

Figure 4 indicates that even in the case of $\operatorname{opt}_{k}(G, \mathcal{W})=\left\lceil\alpha_{k}(G, \mathcal{W}) / 2\right\rceil$, a greedy splitting in Step 2 may not construct an optimal solution unless hooking up operations are used. To justify the algorithm NAEC-AUG, it suffices to show the following theorem.

Theorem 5.1 Let $(G, \mathcal{W})$ and $H$ satisfy the assumption of Theorem 4.2, and $k \geq 3$ be an integer. Let $H^{*}$ be a graph obtained by a sequence of admissible splittings at $s$ from $H$ such that $E_{H^{*}}(s, V) \neq \emptyset$ holds and no pair of two edge in $E_{H^{*}}(s, V)$ is admissible in $H^{*}$. Let $C_{1}$ and $C_{2}$ be two components in $H^{*}-s$ with $d_{H^{*}}\left(s, C_{1}\right)=3$ and $d_{H^{*}}\left(s, C_{2}\right)=1$ (they exist by Theorem 4.2). 
Figure 4: Illustration of a graph $H=\left(V \cup\{s\}, E \cup F_{1}\right)$ satisfying (4.1) for $k=3$, where edges in $F_{1}$ are drawn by broken lines. If we first execute the admissible splitting of $\left(s, v_{2}\right)$ and $\left(s, v_{3}\right)$, then a complete splitting can be found. However, the resulting graph $H_{1}=(H-$ $\left.\left\{\left(s, v_{1}\right),\left(s, v_{2}\right)\right\}\right) \cup\left\{\left(v_{1}, v_{2}\right)\right\}$ obtained from $H$ by the admissible splitting of $\left(s, v_{1}\right)$ and $\left(s, v_{2}\right)$ has no admissible splitting pair at $s$.

Then if $H^{*}$ satisfies one of the following conditions $(a)-(c)$, then $H$ has a complete admissible splitting at $s$ after replacing at most one edge in $E_{H}(s, V)$. Otherwise $(G, \mathcal{W})$ has property $(\mathrm{P})$. (a) For $\left\{\left(s, u^{*}\right)\right\}=E_{H^{*}}\left(s, C_{2}\right), u^{*}$ is contained in no cut $X \subseteq C_{2}$ of type $(A)$ with $d_{H^{*}}(X)=k$. (b) $E_{1} \cap E\left(H^{*}\left[V-C_{1}\right]\right) \neq \emptyset$ holds, where $E_{1}$ denotes the set of all split edges.

(c) There is a set $E^{\prime} \subseteq E_{1}$ of at most two split edges such that the graph $H^{\prime}$ resulting from hooking up the set $E^{\prime}$ of edges in $H^{*}$ has an admissible pair $\left\{\left(s, u^{*}\right), f\right\}$ for some $f \in E_{H^{\prime}}(s, V)$.

Proof: See Section 6.

By Theorems 4.2 and 5.1, for the set $E^{*}$ of edges obtained by algorithm NAEC-AUG, the graph $H^{*}=\left(V \cup\{s\}, E \cup E^{*}\right)$ satisfies (4.1), i.e., $d_{H^{*}}(X) \geq k$ for all cuts $X \subset V$ of type (A) or (B). By $d_{H^{*}}(s)=0$, we have $d_{G+E^{*}}(X)=d_{H^{*}}(X) \geq k$ for all cuts $X \subset V$ of type (A) or (B). This implies that the graph $G+E^{*}$ is $k$-NA-edge-connected. By Theorems 4.1 and 5.1, we have $\left|E^{*}\right|=\left\lceil\alpha_{k}(G, \mathcal{W}) / 2\right\rceil+1$ in the cases where an initial area graph $(G, \mathcal{W})$ has property $(\mathrm{P})$, $\left|E^{*}\right|=\left\lceil\alpha_{k}(G, \mathcal{W}) / 2\right\rceil$ otherwise. By Lemmas 3.2 and 3.4 , we have $\left|E^{*}\right|=\operatorname{opt}_{k}(G, \mathcal{W})$.

We analyze the time complexity of algorithm NAEC-AUG. It is not difficult to verify that it can be computed in polynomial time. In Step 1, we first add $k$ edges between $s$ and $V$ so that $H$ satisfies (4.1) and we attain $F_{1}$ minimal with respect to (4.1) as follows. For each vertex $v \in V$, after deleting all edges between $s$ and $v$, we check whether the resulting graph $H^{\prime}$ satisfies (4.1) or not. By regarding $k$ multiple edges as one edge with capacity $k$ and using the maximum flow technique, we can compute in polynomial time $\lambda_{H^{\prime}}(v, W)$ for a vertex $v \in V$ and an area $W \in \mathcal{W}$; we can check in polynomial time whether $H^{\prime}$ satisfies (4.1) or not. If (4.1) is violated, then we add $\max _{x \in V, W \in \mathcal{W}}\left\{k-\lambda_{H^{\prime}}(x, W)\right\}$ edges between $s$ and $v$ in $H^{\prime}$. In 


\section{Proof of Theorem 5.1}

We first show the following property of minimal dangerous cuts.

Lemma 6.1 Let $H=(V \cup\{s\}, E \cup F)$ be a k-extension of $(G=(V, E), \mathcal{W})$ and $\{(s, u),(s, v)\}$ be a pair of edges in $F$ which is not admissible. Let $Y \subset V$ be a dangerous cut of type $(B)$ with $\{u, v\} \subseteq Y$ such that no $Y^{\prime} \subset Y$ with $\{u, v\} \subseteq Y^{\prime}$ is a dangerous cut of type $(B)$. Then $Y$ is unique.

Proof: Assume by contradiction that there are two distinct dangerous cuts $Y_{1}$ and $Y_{2}$ of type (B) with $\{u, v\} \subseteq Y_{1} \cap Y_{2}$ such that no $Y^{\prime} \subset Y_{i}$ with $\{u, v\} \subseteq Y^{\prime}$ is a dangerous cut of type $(B)$ for each $i=1,2$. From the minimality of $Y_{i}, Y_{1}$ and $Y_{2}$ cross each other in $H$. By Lemma 4.6, we have $d_{H}\left(s, Y_{1} \cap Y_{2}\right)=1$, contradicting $\{u, v\} \subseteq Y_{1} \cap Y_{2}$.

In this section, let $G, H$, and $H^{*}$ satisfy the assumption of Theorem $5.1, E_{1}$ be the set of all split edges in $H^{*}$, and $F^{*}=E_{H^{*}}(s, V)$ (note $H^{*}=\left(V \cup\{s\}, E \cup E_{1} \cup F^{*}\right)$ ). Since $H^{*}$ has no admissible splitting pair at $s$, Theorem 4.2 implies that the graph $G^{*}=H^{*}-s$ has two components $C_{1}$ and $C_{2}$ with $d_{H^{*}}\left(s, C_{1}\right)=3$ and $d_{H^{*}}\left(s, C_{2}\right)=1$. Let $V\left[F^{*}\right] \cap C_{1}=\left\{u_{0}, u_{1}, u_{2}\right\}$ and $V\left[F^{*}\right] \cap C_{2}=\left\{u_{3}=u^{*}\right\}$. An outline of the proof of Theorem 5.1 is given as follows. We first show that if at least one of the conditions (a) - (c) in Theorem 5.1 hold, then $H^{*}$ can be modified to a graph $H^{\prime}$ by replacing or hooking up edges in $F^{*} \cup E_{1}$ so that $H^{\prime}$ has a complete admissible splitting. We next show that if none of $(\mathrm{a})-(\mathrm{c})$ holds, then $(G, \mathcal{W})$ has property $(\mathrm{P})$.

For a $k$-extension $H=(V \cup\{s\}, E \cup F)$ of $(G=(V, E), \mathcal{W})$, a cut $X \subset V$ is called critical if $X$ is of type (A) or (B) and we have $d_{H}(X)=k$. The next lemma shows the cases where (a) holds. 
Lemma 6.2 Let $k \geq 2$. If $u_{3}$ is contained in no critical cut of type $(A)$ in $H^{*}$, then after replacing the edge $\left(s, u_{3}\right)$ with a new edge $(s, x)$ for some vertex $x \in C_{1}$, we can continue admissible edge-splittings until isolating s.

Proof: Assume that $u_{3}$ is contained in no critical cut of type $(A)$ in $H^{*}$. Then $u_{3}$ is contained in a critical cut of type $(B)$ or no critical cut. Let $X_{u}$ denote a critical cut of type (B) with $u_{3} \in$ $X_{u} \subset V$ such that no cut $X^{\prime} \subset X_{u}$ with $u_{3} \in X^{\prime}$ is critical of type (B) if exists, $X_{u}=V$ otherwise. Then $X_{u} \cap C_{1} \neq \emptyset$ holds since otherwise $V-C_{1}$ is of type (B) and hence $d_{H^{*}}\left(V-C_{1}\right) \geq k \geq 2$ holds by (4.1), contradicting $d_{H^{*}}\left(V-C_{1}\right)=d_{H^{*}}\left(s, C_{2}\right)=1$. Let $H_{1}=\left(H^{*}-\left\{\left(s, u_{3}\right)\right\}\right) \cup\{(s, x)\}$ be the graph obtained from replacing the edge $\left(s, u_{3}\right)$ with $(s, x)$ with some $x \in X_{u} \cap C_{1}$ in $H^{*}$.

We claim that $H_{1}$ also satisfies (4.1). Assume by contradiction that $H_{1}$ violates (4.1). Then $H^{*}$ has a critical cut $X^{\prime} \subset V$ with $u_{3} \in X^{\prime} \cap X_{u}$ and $x \in X_{u}-X^{\prime}$. Note that $X^{\prime}$ is of type (B) from the assumption of $u_{3}$. We have $X^{\prime}-X_{u} \neq \emptyset$ from the minimality of $X_{u}$ and hence $X_{u}$ and $X^{\prime}$ cross each other in $H^{*}$. Now $X_{u}-X^{\prime}$ (resp., $X^{\prime}-X_{u}$ ) is of type (A) since it is disjoint with an area included in $X^{\prime}$ (resp., $X_{u}$ ) (note that both of $X^{\prime}$ and $X_{u}$ are of type (B)). By (4.1), we have $d_{H^{*}}\left(X_{u}-X^{\prime}\right) \geq k$ and $d_{H^{*}}\left(X^{\prime}-X_{u}\right) \geq k$. From $d_{H^{*}}\left(X_{u}\right)=d_{H^{*}}\left(X^{\prime}\right)=k$ and $(2.1)$, we have $d_{H^{*}}\left(s, X_{u} \cap X^{\prime}\right)=0$, contradicting $u_{3} \in X_{u} \cap X^{\prime}$.

All neighbors of $s$ in $H_{1}$ are contained in a component in $H_{1}[V]$, and hence we can continue admissible edge-splittings until isolating $s$ in $H_{1}$ by Theorem 4.2.

Next we show the cases where (b) or (c) hold in the following Lemmas 6.4 and 6.5, respectively. For $\left(s, u_{0}\right) \in F^{*}$, none of $\left\{\left(s, u_{0}\right),\left(s, u_{i}\right)\right\}$ for $i=1,2,3$ is admissible in $H^{*}$. Hence Theorem 4.8 implies that $H^{*}$ has two dangerous cuts $Y_{1}$ and $Y_{2}$ of type (A) with $C_{1}=Y_{1} \cup Y_{2}$ and $\left\{\left(s, u_{0}\right),\left(s, u_{i}\right)\right\}=E_{H^{*}}\left(s, Y_{i}\right)$ for $i=1,2$. By Lemma 4.6, we have $d_{H^{*}}\left(Y_{1}-Y_{2}\right)=d_{H^{*}}\left(Y_{2}-Y_{1}\right)=$ $k$. Moreover, the graph $H^{*}$ has the following property.

Lemma 6.3 Let $k \geq 3 . \lambda\left(G^{*}\left[C_{1}\right]\right) \geq 2$ holds.

Proof: $G^{*}\left[C_{1}\right]$ is connected and so assume by contradiction that $\lambda\left(G^{*}\left[C_{1}\right]\right)=1$, i.e., there is a cut $X \subset C_{1}$ with $d_{G^{*}}\left(X, C_{1}-X\right)=1$. Let $d_{H^{*}}(s, X) \leq 1$ without loss of generality (note that $d_{H^{*}}\left(s, C_{1}\right)=3$ holds). Since $Y_{1}-Y_{2}$ is a cut of type (A) with $d_{H^{*}}\left(Y_{1}-Y_{2}\right)=k$, we have $\lambda\left(G^{*}\left[Y_{1}-Y_{2}\right]\right) \geq 2$ by Lemma 4.5. Similarly, $\lambda\left(G^{*}\left[Y_{2}-Y_{1}\right]\right) \geq 2$ holds. Hence $X$ cannot cross with any of $Y_{1}-Y_{2}$ and $Y_{2}-Y_{1}$. Moreover, by $d_{H^{*}}(s, X) \leq 1, X$ can contain at most one of $Y_{1}-Y_{2}$ and $Y_{2}-Y_{1}$. This implies that $X \subseteq Y_{1}$ or $X \subseteq Y_{2}$ holds, contradicting that any cut $X^{\prime}$ of type (A) must satisfy $d_{H^{*}}\left(X^{\prime}\right) \geq k \geq 3$.

Lemma 6.4 Let $k \geq 3$. If $E_{1} \cap E\left(G^{*}\left[V-C_{1}\right]\right) \neq \emptyset$ holds, then after hooking up one edge in $E_{1} \cap E\left(G^{*}\left[V-C_{1}\right]\right)$, we can continue admissible edge-splittings until isolating s. 
Proof: Let $e_{1}=(x, y) \in E_{1} \cap E\left(G^{*}\left[V-C_{1}\right]\right)$ and $H_{1}=\left(H^{*}-\{(x, y)\}\right) \cup\{(s, x),(s, y)\}$ be the graph obtained from hooking up $e_{1}$ in $H^{*}$. We claim that

$$
\left\{\left(s, u_{0}\right), f\right\} \text { is admissible in } H_{1} \text { for some edge } f=(s, z) \text { with } z \in\left\{u_{3}, x, y\right\} \text {. }
$$

Note that also in $H_{1}$, neither $\left\{\left(s, u_{0}\right),\left(s, u_{1}\right)\right\}$ nor $\left\{\left(s, u_{0}\right),\left(s, u_{2}\right)\right\}$ is admissible. Assume by contradiction that (6.1) does not hold. Then there is no edge $f^{\prime} \in E_{H_{1}}(s, V)$ such that $\left\{f^{\prime},\left(s, u_{0}\right)\right\}$ is admissible in $H_{1}$. By $d_{H_{1}}\left(s, C_{1}\right) \geq 3$, the statement (ii) in Theorem 4.8 holds for the edge $\left(s, u_{0}\right)$. This and Lemma 4.5 imply that all neighbors of $s$ except exactly one neighbor are contained in a single component in $H_{1}[V]$, a contradiction. Therefore (6.1) holds.

Let $H_{2}$ be the graph obtained from splitting $\left\{\left(s, u_{0}\right), f\right\}$ in $H_{1}$. We here claim that there is a sequence of admissible edge-splittings in $H_{2}$ until the vertex $s$ is isolated. Otherwise Theorem 4.2 says that $H_{2}[V]$ has two components $C_{1}^{\prime}, C_{2}^{\prime} \subset V$ with $d_{H_{2}}\left(s, C_{1}^{\prime}\right)=3$ and $d_{H_{2}}\left(s, C_{2}^{\prime}\right)=1$. By $k \geq 3$ and Lemma 6.3 , we have $\lambda\left(H_{2}\left[C_{1}^{\prime}\right]\right) \geq 2$ (note that $d_{H_{2}}(s)=4$ holds). Clearly, $\left\{u_{0}, u_{1}, u_{2}, z\right\} \subseteq C_{1}^{\prime}$ hold. However, the edge $\left(u_{0}, z\right)$ is a bridge in $H_{2}\left[C_{1}^{\prime}\right]$, a contradiction.

Lemma 6.5 Let $k \geq 3$ and $E_{1} \cap E\left(G^{*}\left[C_{1}\right]\right) \neq \emptyset$. Assume that there is a set $E^{\prime}$ of at most two split edges in $E_{1} \cap E\left(G^{*}\left[C_{1}\right]\right)$ in $H^{*}$ such that the graph $H_{1}$ resulting from hooking up all edges in $E^{\prime}$ in $H^{*}$ has an admissible pair $\left\{f,\left(s, u_{3}\right)\right\}$ for some edge $f \in E_{H_{1}}(s, V)$. Then in $H_{1}$, we can continue admissible edge-splittings until isolating s.

Proof: Let $E_{1}^{\prime} \subseteq E_{1} \cap E\left(G^{*}\left[C_{1}\right]\right)$ denote a set of split edges with $\left|E_{1}^{\prime}\right| \leq 2$ such that $\{f=$ $\left.(s, x),\left(s, u_{3}\right)\right\}$ is admissible for some $f \in E_{H_{1}}(s, V)$ in $H_{1}$, where $H_{1}$ results from hooking up all edges in $E_{1}^{\prime}$ in $H^{*}$. Let $H_{2}=\left(H_{1}-\left\{(s, x),\left(s, u_{3}\right)\right\}\right) \cup\left\{\left(x, u_{3}\right)\right\}$. If $H_{1}\left[C_{1}\right]$ is connected, then all neighbors of $s$ in $H_{2}$ are contained in a component in $H_{2}[V]$, and hence Theorem 4.2 implies that we can execute a complete splitting at $s$ in $H_{2}$ while preserving (4.1).

We consider the case where $H_{1}\left[C_{1}\right]$ is not connected. By Lemma $6.3, \lambda\left(G^{*}\left[C_{1}\right]\right) \geq 2$ holds. Hence we have $\left|E_{1}^{\prime}\right|=2$ and the number of components in $H_{1}\left[C_{1}\right]$ is two. Let $C_{1}^{\prime}, C_{2}^{\prime}$ be components in $H_{1}\left[C_{1}\right]$ with $d_{H_{1}}\left(s, C_{1}^{\prime}\right) \geq d_{H_{1}}\left(s, C_{2}^{\prime}\right)$ without loss of generality (note that $E_{1}^{\prime}=$ $E_{G^{*}}\left(C_{1}^{\prime}, C_{2}^{\prime}\right)$ holds). Then by $d_{H^{*}}\left(s, C_{1}\right)=3$, we have the following two possible cases (I) and (II). (I) $d_{H_{1}}\left(s, C_{1}^{\prime}\right)=5$ and $d_{H_{1}}\left(s, C_{2}^{\prime}\right)=2$ and (II) $d_{H_{1}}\left(s, C_{1}^{\prime}\right)=4$ and $d_{H_{1}}\left(s, C_{2}^{\prime}\right)=3$.

In both cases of (I) and (II), all neighbors of $s$ in $H_{2}$ are contained in at most two components in $H_{2}[V]$ from the construction of $H_{2}$. By $d_{H_{2}}(s)=6$, Theorem 4.2 says that $H_{2}$ always has an admissible pair $\left\{\left(s, v^{\prime}\right),\left(s, v^{\prime \prime}\right)\right\} \subseteq E_{H_{2}}(s, V)$. Let $H_{3}$ denote the graph obtained from splitting $\left(s, v^{\prime}\right)$ and $\left(s, v^{\prime \prime}\right)$ in $H_{2}$. If $H_{3}$ also has an admissible pair at $s$, then the lemma is proved. Otherwise Theorem 4.2 says that $H_{3}[V]$ has two components $C_{1}^{*}$ and $C_{2}^{*}$ with $d_{H_{3}}\left(s, C_{1}^{*}\right)=3$, and $d_{H_{3}}\left(s, C_{2}^{*}\right)=1$. Then in both cases of (I) and (II), $E\left(H_{3}\left[C_{2}^{*}\right]\right)$ contains the split edges $f^{*}$ with $f^{*}=\left(x, u_{3}\right)$ or $f^{*}=\left(v^{\prime}, v^{\prime \prime}\right)$. Lemma 6.4 implies that after hooking up the edge $f^{*}$, we can execute a complete splitting at $s$ while preserving (4.1), which proves the lemma. 
We finally show that if none of $(\mathrm{a})-(\mathrm{c})$ holds, then $(G, \mathcal{W})$ has property $(\mathrm{P})$ by the following two lemmas.

Lemma 6.6 Let $H^{*}$ satisfy none of $(a)-(c)$ in Theorem 5.1. Then for every vertex $v \in$ $V\left[E_{1}\right] \cup\left(V\left[F^{*}\right]-\{s\}\right)$, there is a cut $X_{v} \subset V$ with $v \in X_{v}$ satisfying the following $(i)$ and (ii). (i) $d_{H^{*}}\left(X_{v}\right)=k$ and $X_{v}$ is of type $(A)$.

(ii) If $v \in C_{1}$ holds, then there is a cut $Y_{v} \supseteq X_{v}$ of type $(B)$ with $u_{3} \in Y_{v}, d_{H^{*}}\left(Y_{v}\right) \leq k+1$, and $E\left(G^{*}\left[Y_{v}\right]\right) \cap E_{1}=\emptyset$.

Proof: Since (a) does not hold, the lemma for $v=u_{3}$ holds. Since (b) does not hold, we have $V\left[E_{1}\right] \cup\left(V\left[F^{*}\right]-\left\{s, u_{3}\right\}\right) \subseteq C_{1}$. We first show the lemma for vertices in $V\left[F^{*}\right]-\left\{s, u_{3}\right\}$ and next show it for vertices in $V\left[E_{1}\right]$.

Let $Y_{i}^{\prime}$ be a dangerous cut containing two vertices $u_{i}$ and $u_{3}$ in $H^{*}$ such that no cut $Y^{\prime \prime} \subset Y_{i}^{\prime}$ with $\left\{u_{i}, u_{3}\right\} \subseteq Y^{\prime \prime}$ is dangerous in $H^{*}$ for $i=0,1,2$. Lemma 6.1 says that $Y_{i}^{\prime}$ is unique (note that any dangerous cut $Y$ containing $u_{3}$ and a vertex in $C_{1}$ is of type (B) since $H^{*}[Y]$ is disconnected). By Lemma 4.3, we have $d_{H^{*}}\left(s, V-Y_{i}^{\prime}\right) \geq d_{H^{*}}\left(s, Y_{i}^{\prime}\right)-1$ and hence $Y_{i}^{\prime} \cap\left\{u_{0}, u_{1}, u_{2}\right\}=\left\{u_{i}\right\}$. Hence for any pair $\{i, j\} \subset\{0,1,2\}$ with $i \neq j, Y_{i}^{\prime}$ and $Y_{j}^{\prime}$ cross each other in $H^{*}$. By Lemma 4.6, both of $Y_{i}^{\prime}-Y_{j}^{\prime}$ and $Y_{j}^{\prime}-Y_{i}^{\prime}$ are of type (A) and we have $d_{H^{*}}\left(Y_{i}^{\prime} \cap Y_{j}^{\prime}, V \cup\{s\}-Y_{i}^{\prime}-Y_{j}^{\prime}\right)=d_{H^{*}}\left(s, u_{3}\right)=1$,

$$
d_{H^{*}}\left(Y_{i}^{\prime}-Y_{j}^{\prime}\right)=d_{H^{*}}\left(Y_{j}^{\prime}-Y_{i}^{\prime}\right)=k, u_{i} \in Y_{i}^{\prime}-Y_{j}^{\prime}, \text { and } u_{j} \in Y_{j}^{\prime}-Y_{i}^{\prime}
$$

Then we claim that

$$
E\left(G^{*}\left[Y_{i}^{\prime}\right]\right) \cap E_{1}=\emptyset
$$

holds for $i=0,1,2$. Assume by contradiction that $E\left(G^{*}\left[Y_{i}^{\prime}\right]\right) \cap E_{1} \neq \emptyset$ holds. Let $\left(y_{1}, y_{2}\right) \in$ $E\left(G^{*}\left[Y_{i}^{\prime}\right]\right) \cap E_{1}$ and $H_{1}=\left(H^{*}-\left\{\left(y_{1}, y_{2}\right)\right\}\right) \cup\left\{\left(s, y_{1}\right),\left(s, y_{2}\right)\right\}$ be the graph obtained by hooking up the edge $\left(y_{1}, y_{2}\right)$ in $H^{*}$. Then $\left\{\left(s, u_{i}\right),\left(s, u_{3}\right)\right\}$ is admissible in $H_{1}$, because any cut $Y$ with $\left\{u_{i}, u_{3}\right\} \subseteq Y$ which is dangerous in $H^{*}$ satisfies $Y \supseteq Y_{i}^{\prime}$ by the minimality and uniqueness of $Y_{i}^{\prime}$ and hence $d_{H_{1}}(Y)=d_{H^{*}}(Y)+2$, and hence it is non-dangerous in $H_{1}$. This contradicts that $H^{*}$ does not satisfy (c). Hence (6.2) holds for each $i=0,1,2$. Therefore the cuts $X_{u_{i}}=Y_{i}^{\prime}-Y_{j}^{\prime}$ and $Y_{u_{i}}=Y_{i}^{\prime}$ for $i=0,1,2$ prove the lemma for any vertex $v \in V\left[F^{*}\right]-\left\{s, u_{3}\right\}$.

Finally, we prove the lemma for any vertex $v \in V\left[E_{1}\right]$. Let $\left(x_{1}, x_{2}\right) \in E_{1}$ and $H_{2}=\left(H^{*}-\right.$ $\left.\left\{\left(x_{1}, x_{2}\right)\right\}\right) \cup\left\{\left(s, x_{1}\right),\left(s, x_{2}\right)\right\}$ be the graph obtained by hooking up the edge $\left(x_{1}, x_{2}\right)$ in $H^{*}$. Since (c) does not hold, neither $\left\{\left(s, u_{3}\right),\left(s, x_{1}\right)\right\}$ nor $\left\{\left(s, u_{3}\right),\left(s, x_{2}\right)\right\}$ is admissible in $H_{2}$. Let $Y_{i}^{\prime} \subset V$ denote a dangerous cut with $\left\{u_{3}, x_{i}\right\} \subseteq Y_{i}^{\prime}$ in $H_{2}$ such that no $Y^{\prime \prime} \subset Y_{i}^{\prime}$ with $\left\{u_{3}, x_{i}\right\} \subseteq Y^{\prime \prime}$ is dangerous in $H_{2}$ for $i=1,2$. Lemma 6.1 says that $Y_{i}^{\prime}$ is unique. Since $\left\{\left(s, x_{1}\right),\left(s, x_{2}\right)\right\}$ is clearly admissible in $H_{2}$, we have $x_{1} \in Y_{1}^{\prime}-Y_{2}^{\prime}$ and $x_{2} \in Y_{2}^{\prime}-Y_{1}^{\prime}$, and $Y_{1}^{\prime}$ and $Y_{2}^{\prime}$ cross each other in $H_{2}$. By Lemma 4.6, both of $Y_{1}^{\prime}-Y_{2}^{\prime}$ and $Y_{2}^{\prime}-Y_{1}^{\prime}$ are of type (A) and we have 


$$
\begin{aligned}
& d_{H_{2}}\left(Y_{1}^{\prime} \cap Y_{2}^{\prime}, V \cup\{s\}-Y_{1}^{\prime}-Y_{2}^{\prime}\right)=d_{H_{2}}\left(s, u_{3}\right)=1 \text { and } \\
& d_{H_{2}}\left(Y_{1}^{\prime}\right)=d_{H^{*}}\left(Y_{1}^{\prime}\right), d_{H_{2}}\left(Y_{1}^{\prime}-Y_{2}^{\prime}\right)=d_{H^{*}}\left(Y_{1}^{\prime}-Y_{2}^{\prime}\right)=k, x_{1} \in Y_{1}^{\prime}-Y_{2}^{\prime}, \\
& d_{H_{2}}\left(Y_{2}^{\prime}\right)=d_{H^{*}}\left(Y_{2}^{\prime}\right), d_{H_{2}}\left(Y_{2}^{\prime}-Y_{1}^{\prime}\right)=d_{H^{*}}\left(Y_{2}^{\prime}-Y_{1}^{\prime}\right)=k, x_{2} \in Y_{2}^{\prime}-Y_{1}^{\prime} .
\end{aligned}
$$

Moreover, similarly to the arguments about vertices in $V\left[F^{*}\right]-\left\{s, u_{3}\right\}$, by using the assumption that $(c)$ does not hold, we see that $E\left(G^{*}\left[Y_{i}^{\prime}\right]\right) \cap E_{1}=\emptyset$ holds for $i=1,2$. Therefore the cuts $X_{x_{i}}=Y_{i}^{\prime}-Y_{j}^{\prime}$ and $Y_{x_{i}}=Y_{i}^{\prime}$ for $\{i, j\}=\{1,2\}$ prove the lemma for any vertex $v \in V\left[E_{1}\right]$.

Lemma 6.7 Let $H^{*}$ satisfy none of $(a)-(c)$ in Theorem 5.1. Then $G$ has property $(\mathrm{P})$.

Proof: By Lemma 6.6, for any $v \in V\left[E_{1}\right] \cup\left(V\left[F^{*}\right]-s\right)$, there are two cuts $X_{v}$ and $Y_{v}$ of Lemma 6.6 (i) and (ii). Then let $X_{v}$ be a cut such that no cut $X^{\prime} \subset X_{v}$ with $v \in X^{\prime}$ satisfies this property. Moreover, we can assume that $Y_{v} \supseteq V-C_{1}$ holds. This follows since for any cut $Y_{v}$ satisfying Lemma 6.6(ii), we have $X_{v} \subseteq Y_{v} \cup\left(V-C_{1}\right), d_{H^{*}}\left(Y_{v} \cup\left(V-C_{1}\right)\right) \leq d_{H^{*}}\left(Y_{v}\right) \leq k+1$ (by $d_{H^{*}}\left(V-C_{1}\right)=d_{H^{*}}\left(s, u_{3}\right)=1$ and $\left.u_{3} \in Y_{v}\right)$, and $E\left(G^{*}\left[Y_{v} \cup\left(V-C_{1}\right)\right]\right) \cap E_{1}=\emptyset$ (since (b) does not hold), which implies that the cut $Y_{v} \cup\left(V-C_{1}\right)$ also satisfies Lemma 6.6(ii). Let $\mathcal{X}$ be the family of all cuts $X_{v}, v \in V\left[E_{1}\right] \cup\left(V\left[F^{*}\right]-s\right)$ such that $\bigcup_{X \in \mathcal{X}} X$ includes $V\left[E_{1}\right] \cup\left(V\left[F^{*}\right]-s\right)$ and no cut $X_{v} \in \mathcal{X}$ satisfies $X_{v} \subset X$ for some $X \in \mathcal{X}$, and $\mathcal{Y}$ be the family of the corresponding cuts $Y_{v}$. We will show that $\alpha_{k}(G, \mathcal{W})$ is even and the family $\mathcal{X}$ is a subpartition of $V$ satisfying $\sum_{X \in \mathcal{X}}\left(k-d_{G}(X)\right)=\alpha_{k}(G, \mathcal{W})$ and $(\mathrm{P} 1)-(\mathrm{P} 3)$.

We claim that

$$
\mathcal{X} \text { is a subpartition of } V \text {. }
$$

Otherwise there are two cuts $X_{u}, X_{v} \in \mathcal{X}$ which cross each other in $H^{*}$. Since both of $X_{u}$ and $X_{v}$ are of type (A), by (4.1) and (4.2), we have $d_{H^{*}}\left(X_{u}-X_{v}\right) \geq k$ and $d_{H^{*}}\left(X_{v}-X_{u}\right) \geq k$. From $d_{H^{*}}\left(X_{u}\right)=d_{H^{*}}\left(X_{v}\right)=k$ and $(2.1)$, we have $d_{H^{*}}\left(X_{u}-X_{v}\right)=d_{H^{*}}\left(X_{v}-X_{u}\right)=k$ and $d_{H^{*}}\left(X_{u} \cap X_{v},(V \cup\{s\})-X_{u}-X_{v}\right)=0$. Then $u \in X_{u}-X_{v}$ holds, since if $u \in X_{u} \cap X_{v}$ holds, then there is a split edge $\left(u, u^{\prime}\right) \in E_{1}$ with $u^{\prime} \in X_{u} \cup X_{v}$ by $d_{H^{*}}\left(X_{u} \cap X_{v},(V \cup\{s\})-X_{u}-X_{v}\right)=0$, which contradicts $E\left(G^{*}\left[X_{u}\right]\right) \cap E_{1}=\emptyset=E\left(G^{*}\left[X_{v}\right]\right) \cap E_{1}$. From $d_{H^{*}}\left(X_{u}-X_{v}\right)=k$ and $u \in X_{u}-X_{v}$, the cut $X_{u}-X_{v}$ contradicts the minimality of $X_{u}$.

By (6.3) and $E\left(G^{*}[X]\right) \cap E_{1}=\emptyset$ for every $X \in \mathcal{X}$, we have $\sum_{X \in \mathcal{X}}\left(k-d_{G}(X)\right)=\alpha_{k}(G, \mathcal{W})$, and $\alpha_{k}(G, \mathcal{W})$ is even by $\alpha_{k}(G, \mathcal{W})=2\left|E_{1}\right|+\left|F^{*}\right|$. Moreover, $\mathcal{X}$ is a subpartition of $V$ satisfying $(\mathrm{P} 1)$ and (P2) by taking $X_{1}=X_{u_{3}}$. Now for every $Y \in \mathcal{Y}$ which does not cross with any $X_{i} \in \mathcal{X}$ in $H^{*}$, we have $\sum_{X^{\prime} \in \mathcal{X}, X^{\prime} \subseteq Y}\left(k-d_{G}\left(X^{\prime}\right)\right) \leq(k+1)-d_{G}(Y)$ by $E\left(G^{*}[Y]\right) \cap E_{1}=\emptyset$. Therefore, in order to show that $\mathcal{X}$ satisfies (P3), we finally prove that for any $X_{u} \in \mathcal{X}$ with $u \neq u_{3}$, there is a cut $Y_{w} \in \mathcal{Y}$ with $X_{u} \subseteq Y_{w}$ such that for any cut $X \in \mathcal{X}, Y_{w}$ and $X$ do not cross each other 


\section{Conclusion}

In this paper, we considered the problem of asking to augment a given area graph $(G=$ $(V, E), \mathcal{W})$ by adding the minimum number of new edges such that the resulting graph becomes $k$-NA-edge-connected. We showed that the problem in the case of $k \geq 3$ can be solved in polynomial time. The time complexity of our algorithm is $O\left(m+n\left(k^{3}+n^{2}\right)(p+k n+n \log n) \log k+\right.$ $\left.p k n^{3} \log (n / k)\right)$, where $n=|V|, m=|\{\{u, v\} \mid(u, v) \in E\}|$, and $p=|\mathcal{W}|$. This paper treated the cases where the connectivity requirement between a vertex $v \in V$ and an area $W \in \mathcal{W}$ is unique. It is a future work to consider the problems with general connectivity requirements.

\section{Acknowledgments:}

We are very grateful to the anonymous referee for his careful readings and suggestions. This research was partially supported by the Grant-in-Aid for Scientific Research from the Ministry of Education, Culture, Sports, Science and Technology.

\section{References}

[1] K. Arata, S. Iwata, K. Makino, and S. Fujishige, Locating sources to meet flow demands in undirected networks, J. Algorithms, 42, (2002), 54-68. 
[2] A. Benczúr and A. Frank, Covering symmetric supermodular function by graphs, Mathematical Programming, 84, (1999), 483-503.

[3] J. Bang-Jensen, H. N. Gabow, T. Jordán and Z. Szigeti, Edge-connectivity augmentation with partition constraints, SIAM J. Discrete Math., 12, (1999), 160-207.

[4] M. Garey and D. Johnson, Computers and Intractability, W. H. Freeman and Company, San Francisco, CA, 1979.

[5] A. Frank, Augmenting graphs to meet edge-connectivity requirements, SIAM J. Discrete Math., 5(1), (1992), 25-53.

[6] A. Frank, Connectivity augmentation problems in network design, in Mathematical Programming: State of the Art 1994, J.R. Birge and K.G. Murty (Eds.), The University of Michigan, Ann Arbor, MI, (1994), 34-63.

[7] H. N. Gabow, Applications of a poset representation to edge connectivity and graph rigidity, Proc. 32nd IEEE Symp. Found. Comp. Sci., (1991), 812-821.

[8] M. Grötschel, C. L. Monma and M. Stoer, Design of survivable networks, in: Handbook in Operations Research and Management Science, 7, Network Models, North-Holland, Amsterdam, (1995), 617-672.

[9] H. Ito, Node-to-area connectivity of graphs, Transactions of the Institute of Electrical Engineers of Japan, 11C(4), (1994), 463-469.

[10] H. Ito, Node-to-area connectivity of graphs, In M. Fushimi and K. Tone, editors, Proceedings of APORS94, World Scientific publishing, (1995), 89-96.

[11] H. Ito, K. Makino, K. Arata, K. Itatsu, and S. Fujishige, Source location problem with flow requirements in directed networks, Optimization Methods and Software, 18, (2003), $427-435$.

[12] H. Ito and M. Yokoyama, Edge connectivity between nodes and node-subsets, Networks, 31(3), (1998), 157-164.

[13] G. Kant, Algorithms for drawing planar graphs, PhD thesis, Dept. of Computer Science, Utrecht University, the Netherlands, (1993).

[14] M. -Y. Kao, Data security equals graph connectivity, SIAM J. Discrete Math., 9, (1996), $87-100$.

[15] H. Miwa and H. Ito, NA-edge-connectivity augmentation problems by adding edges, Journal of the Operations Research Society of Japan, 47(4), (2004), 224-243. 
[16] H. Nagamochi and T. Ibaraki, A linear-time algorithm for finding a sparse $k$-connected spanning subgraph of a k-connected graph, Algorithmica, 7, (1992), 583-596.

[17] H. Nagamochi and T. Ibaraki, Computing edge-connectivity of multigraphs and capacitated graphs, SIAM J. Discrete Math., 5, (1992), 54-66.

[18] H. Nagamochi and T. Ibaraki, Deterministic $\tilde{O}(\mathrm{~nm})$ time edge-splitting in undirected graphs, J. Combinatorial Optimization, 1, (1997), 5-46.

[19] H. Nagamochi, T. Ishii and H. Ito, Minimum cost source location problem with vertexconnectivity requirements in digraphs, Information Processing Letters, 80(6), (2001), 287294.

[20] Z. Nutov, Approximating connectivity augmentation problems, Proc. the Sixteenth Annual ACM-SIAM Symposium on Discrete Algorithms, (2005), 176-185.

[21] S. Tsukiyama, K. Koike and I. Shirakawa, An algorithm to eliminate all complex triangles in a maximal planar graph for use in VLSI floor-plan, Proc. ISCAS'86, (1986), 321-324.

[22] T. Watanabe and A. Nakamura, Edge-connectivity augmentation problems, J. Comput. System Sci., 35, (1987), 96-144. 